\title{
Scheduling Continuous-Time Kalman Filters
}

\author{
Jerome Le Ny, Member, IEEE, Eric Feron, Member, IEEE, and Munther A. Dahleh, Fellow, IEEE
}

\begin{abstract}
A set of $N$ independent Gaussian linear timeinvariant systems is observed by $M$ sensors whose task is to provide a steady-state causal estimate minimizing the mean-square error on the system states, subject to additional measurement costs. The sensors can switch between systems instantaneously, and there are additional resource constraints, for example on the number of sensors that can observe a given system simultaneously. We first derive a tractable relaxation of the problem, which provides a bound on the achievable performance. This bound can be computed by solving a convex program involving linear matrix inequalities, and moreover this program can be decomposed into coupled smaller dimensional problems. In the scalar case with identical sensors, we give an analytical expression of an index policy proposed in a more general context by Whittle. In the general case, we develop open-loop periodic switching policies whose performance matches the bound arbitrarily closely.
\end{abstract}

Index Terms-Kalman filtering, switched systems, periodic control.

\section{INTRODUCTION}

Advances in sensor networks, networked control systems, and the development of unmanned vehicle systems for intelligence, reconnaissance and surveillance missions require the development of resource allocation algorithms to manage the measurements originating from a large number of sensors observing a large number of targets, see e.g. [1]-[4]. These problems have a long history [5], and include static sensor scheduling problems as well as trajectory optimization scenarios for mobile sensors [4], [6]. In this paper, we consider $M$ sensors tracking the state of $N$ sites or targets in continuous time. The targets can be described by $N$ plants with independent linear time-invariant dynamics

$$
\dot{x}_{i}=A_{i} x_{i}+B_{i} u_{i}+w_{i}, \quad x_{i}(0)=x_{i, 0}, \quad i=1, \ldots, N .
$$

We assume that the plant controls $u_{i}(t)$ are deterministic and known for $t \geq 0$. Each driving noise $w_{i}(t)$ is a stationary white Gaussian noise process with zero mean and known power spectral density matrix $W_{i}$, i.e, $\operatorname{Cov}\left(w_{i}(t), w_{i}\left(t^{\prime}\right)\right)=$ $W_{i} \delta\left(t-t^{\prime}\right), \quad \forall t, t^{\prime}$. The initial conditions are random variables with known mean $\bar{x}_{i, 0}$ and covariance matrices $\Sigma_{i, 0}$. By independent dynamics we mean that the noise processes and initial conditions $x_{i, 0}$ of the different plants are all independent. We also make the following assumption.

Manuscript received November 3, 2008; revised October 6, 2009. Recommended for publication by Associate Editor I. Paschalidis. This work was supported by Air Force - DARPA - MURI award 009628-001-03-132 and Navy ONR award N00014-03-1-0171.

J. Le Ny is with the Department of Electrical and Systems Engineering, University of Pennsylvania, Philadelphia, PA 19104, USA jeromel aseas. upenn. edu.

E. Feron is with the School of Aerospace Engineering, Georgia Tech, Atlanta, GA 30332, USA eric. feron@aerospace. gatech. edu .

M. A. Dahleh is with the Laboratory for Information and Decision Systems, Massachusetts Institute of Technology, Cambridge, MA 02139-4307, USA dahleh@mit.edu.
Assumption 1: The matrices $\Sigma_{i, 0}$ are positive definite for all $i \in\{1, \ldots, N\}$.

This can be achieved by adding an arbitrarily small multiple of the identity matrix to a potentially non-invertible matrix $\Sigma_{i, 0}$. We have $M$ sensors to observe the $N$ plants. If sensor $j$ is used to observe plant $i$, we obtain the linear measurements

$$
y_{i j}=C_{i j} x_{i}+v_{i j} .
$$

Here $v_{i j}$ is a stationary white Gaussian noise process with power spectral density matrix $V_{i j}$, assumed to be positive definite. Also, for simplicity we take $v_{i j}$ to be independent of the other measurement noises, process noises, and initial states. Finally, to guarantee convergence of the filters later on, we make the following assumptions.

Assumption 2: For all $i \in\{1, \ldots, N\}$, the pair $\left(A_{i}, \tilde{C}_{i}\right)$ is detectable, where $\tilde{C}_{i}=\left[C_{i 1}^{T}, \ldots, C_{i M}^{T}\right]^{T}$.

Assumption 3: For all $i \in\{1, \ldots, N\}$, the pair $\left(A_{i}, W_{i}^{1 / 2}\right)$ is controllable.

Let us define $\pi_{i j}(t)=\left\{\begin{array}{l}1 \text { if plant } i \text { is observed at time } t \text { by sensor } j \\ 0 \text { otherwise. }\end{array}\right.$

We assume that each sensor can observe at most one system at each instant, hence we have the constraint

$$
\sum_{i=1}^{N} \pi_{i j}(t) \leq 1, \quad \forall t, \quad j=1, \ldots, M .
$$

If instead sensor $j$ is required to be always operated, constraint (1) should simply be changed to

$$
\sum_{i=1}^{N} \pi_{i j}(t)=1
$$

The equality constraint could be useful in scenarios involving sensors mounted on unmanned vehicles for example, where it might not be possible to withdraw a vehicle from operation during the mission. We also add the following constraint, similar to the one used by Athans [7]. We suppose that each system can be observed by at most one sensor at each instant, so we have

$$
\sum_{j=1}^{M} \pi_{i j}(t) \leq 1, \quad \forall t, \quad i=1, \ldots, N .
$$

Similarly if system $i$ must always be observed by some sensor, constraint (3) can be changed to an equality constraint

$$
\sum_{j=1}^{M} \pi_{i j}(t)=1
$$

Note that a sensor in our discussion can correspond to a combination of several physical sensors, and so the constraints 
above can capture seemingly more general problems where we allow for example more that one measurement per system simultaneously. Using (4) we could also impose a constraint on the total number of allowed observations at each time. Indeed, consider a constraint of the form $\sum_{i=1}^{N} \sum_{j=1}^{M} \pi_{i j}(t) \leq$ $p$, for some positive integer $p$. This constraint means that $M-p$ sensors are required to be idle at each time. To enforce such a requirement, we can create $M-p$ "dummy" systems (simple scalar stable systems to minimize computations), and associate constraint (4) to each of them. Then we simply do not include these systems in the objective function (5) below.

We consider an infinite-horizon average-cost problem. We wish to design an observation policy $\pi(t)=\left\{\pi_{i j}(t)\right\}$ satisfying the constraints (1), (3), or their equality versions, and a causal estimator $\hat{x}_{\pi}$ of $x$, i.e., depending only on the past and current observations produced by the observation policy, such that the average error covariance is minimized, in addition to some sensor utilization costs. The policy $\pi$ itself can also only depend on the past observations. More precisely, we wish to compute

$$
\begin{gathered}
\gamma=\min _{\pi, \hat{x}_{\pi}} \varlimsup_{T \rightarrow \infty} \frac{1}{T} E\left[\int _ { 0 } ^ { T } \sum _ { i = 1 } ^ { N } \left(\left(x_{i}-\hat{x}_{\pi, i}\right)^{\prime} T_{i}\left(x_{i}-\hat{x}_{\pi, i}\right)\right.\right. \\
\left.\left.+\sum_{j=1}^{M} \kappa_{i j} \pi_{i j}(t)\right) d t\right],
\end{gathered}
$$

where the constants $\kappa_{i j} \in \mathbb{R}$ are a cost paid per unit of time when plant $i$ is observed by sensor $j$, the $T_{i}$ 's are positive semidefinite weighting matrices, and $\varlimsup$ lim denotes the upper limit.

Literature review and contributions of this paper. The sensor scheduling problem presented above, except for minor variations, is an infinite horizon version of the problem studied by Athans in [7], who considered the case $N=1$. We include here several plants to show how their independent evolution property can be leveraged in the computations, using the dual decomposition method from optimization. Finitehorizon continuous-time versions of the problem, besides the presentation of Athans [7], have been the subject of several papers [8]-[11]. The solutions proposed involve computational procedures that scale poorly with the dimension of the problem. Discrete-time versions of this sensor selection problem have also received a significant amount of attention, see e.g. [2], [5], [6], [12]-[16]. All algorithms proposed so far for the discrete-time problem, except for the optimal greedy policy in the completely symmetric case [15], either run in exponential time or consist of heuristics with no a priori performance guarantee.

Somewhat surprisingly however, and with the exception of [17], it seems that the infinite-horizon continuous-time version of the Kalman filter scheduling problem has not been considered previously. Mourikis and Roumeliotis [17] also consider initially a discrete-time version of the problem for a particular robotic application. To cope with the difficulty of determining a sensor schedule, they assume instead a model where each sensor can independently take measurements at a constant frequency, and seek the optimal measurement frequencies. In fact, they obtain these frequencies by introducing heuristically a continuous-time Riccati equation, and show that the frequencies can then be computed by solving a semidefinite program. In contrast, we consider the more standard schedulebased version of the problem in continuous time, which is a priori more constraining. We show that essentially the same convex program provides in fact a lower bound on the cost achievable by any measurement policy. We also provide additional insight into the decomposition of the computations of this program, which can be useful in the framework of [17] as well. As demonstrated experimentally in [17], the continuoustime formulation of the problem provides a good heuristic policy even if the filters are implemented digitally, as long as the sampling frequencies are sufficiently fast with respect to the plant dynamics. Moreover, the continuous-time objective (5) has some advantages over purely discrete formulations for sampled-data systems, since these ignore the intersample behavior [18].

The rest of the paper is organized as follows. Section II briefly recalls that for a fixed policy $\pi(t)$, the optimal estimator is obtained by the Kalman-Bucy filter. The properties of the Kalman filter (independence of the error covariance matrix with respect to the measurement values) imply that the remaining problem of finding the optimal scheduling policy $\pi$ is a deterministic control problem. In section III we treat a simplified scalar version of the problem with identical sensors as a special case of the classical "Restless Bandit Problem" (RBP) [19]. We provide analytical expressions for an index policy and for the elements necessary to compute efficiently a lower bound on performance, both of which were proposed in the general setting of the RBP by Whittle. Then, for the multidimensional case treated in full generality in section IV, we show that the lower bound on performance can be computed by solving a convex program involving linear matrix inequalities. This lower bound can be approached arbitrarily closely by a family of new periodically switching policies described in section IV-C. Approaching the bound with these policies is limited only by the frequency with which the sensors can actually switch between the systems. In general, our solution has much more attractive computational properties than the solutions proposed so far for the finite-horizon problem.

\section{OPTIMAL ESTIMATOR}

For a given observation policy $\pi(t)=\left\{\pi_{i j}(t)\right\}_{i, j}$, the minimum variance filter is given by the Kalman-Bucy filter [20], see [7]. The state estimates $\hat{x}_{\pi}$, where the subscript indicates the dependency on the policy $\pi$, are all updated in parallel following the stochastic differential equations

$$
\begin{aligned}
& \frac{d}{d t} \hat{x}_{\pi, i}(t)=A_{i} \hat{x}_{\pi, i}(t)+B_{i}(t) u_{i}(t) \\
& -\Sigma_{\pi, i}(t)\left(\sum_{j=1}^{M} \pi_{i j}(t) C_{i j}^{T} V_{i j}^{-1}\left(C_{i j} \hat{x}_{\pi, i}(t)-y_{i j}(t)\right)\right),
\end{aligned}
$$

with $\hat{x}_{\pi, i}(0)=\bar{x}_{i, 0}$, for $1 \leq i \leq N$. The resulting estimator is unbiased and the error covariance matrix $\Sigma_{\pi, i}(t)$ for system $i$ 
satisfies the matrix Riccati differential equation

$$
\begin{aligned}
\frac{d}{d t} \Sigma_{\pi, i}(t)= & A_{i} \Sigma_{\pi, i}(t)+\Sigma_{\pi, i}(t) A_{i}^{T}+W_{i} \\
& -\Sigma_{\pi, i}(t)\left(\sum_{j=1}^{M} \pi_{i j}(t) C_{i j}^{T} V_{i j}^{-1} C_{i j}\right) \Sigma_{\pi, i}(t)
\end{aligned}
$$

where $\Sigma_{\pi, i}(0)=\Sigma_{i, 0}$. With this result, we can reformulate the optimization of the observation policy as a deterministic optimal control problem. Rewriting $E\left(\left(x_{i}-\hat{x}_{i}\right)^{\prime} T_{i}\left(x_{i}-\hat{x}_{i}\right)\right)=$ $\operatorname{Tr}\left(T_{i} \Sigma_{i}\right)$, the problem (5) asks to compute

$$
\begin{array}{r}
\gamma=\min _{\pi} \varlimsup_{T \rightarrow \infty} \frac{1}{T}\left[\int _ { 0 } ^ { T } \sum _ { i = 1 } ^ { N } \left(\operatorname{Tr}\left(T_{i} \Sigma_{\pi, i}(t)\right)\right.\right. \\
\left.\left.+\sum_{j=1}^{M} \kappa_{i j} \pi_{i j}(t)\right) d t\right],
\end{array}
$$

subject to the constraints (1), (3), or their equality versions, and the dynamics (6).

\section{Plants With Scalar Dynamics and Identical SENSORS}

Before considering the problem in the general multidimensional case, we first assume in this section that

1) the sites or targets have one-dimensional dynamics, i.e., $x_{i} \in \mathbb{R}, i=1, \ldots, N$; and,

2) all the sensors are identical, i.e., $C_{i j}=C_{i}, V_{i j}=$ $V_{i}, \kappa_{i j}=\kappa_{i}, j=1, \ldots, M$.

In this case we can obtain additional insight based on previous work in dynamic scheduling, which also suggests a good policy to follow during the transient regime. We can simplify the problem formulation introduced above so that it corresponds exactly to a special case of the Restless Bandit Problem (RBP) [19]. We define

$$
\pi_{i}(t)=\left\{\begin{array}{l}
1 \text { if plant } i \text { is observed at time } t \text { by a sensor } \\
0 \text { otherwise. }
\end{array}\right.
$$

Since we assumed that a system can be observed by at most one sensor, we can take $M \leq N$. Note that a constraint (4) for some system $i$ can be eliminated, by removing one available sensor that is always measuring system $i$. Constraints (2) and (3) can then be replaced by the single constraint

$$
\sum_{i=1}^{N} \pi_{i}(t)=M, \forall t
$$

This constraint means that at each period, exactly $M$ of the $N$ sites are observed. We treat this case in this section, but again the equality sign can be replaced by an inequality with very little change in our discussion.

To obtain a lower bound on the achievable performance, denoted $\gamma$ in the following, we relax the constraint to enforce it only on average

$$
\varlimsup_{T \rightarrow \infty} \frac{1}{T} \int_{0}^{T} \sum_{i=1}^{N} \pi_{i}(t) d t=M .
$$

Then we adjoin this constraint to the initial objective using a (scalar) Lagrange multiplier $\lambda$ to form the Lagrangian

$$
\begin{array}{r}
L(\pi, \lambda)=\varlimsup_{T \rightarrow \infty} \frac{1}{T} \int_{0}^{T} \sum_{i=1}^{N}\left[\operatorname{Tr}\left(T_{i} \Sigma_{\pi, i}(t)\right)\right. \\
\left.+\left(\kappa_{i}+\lambda\right) \pi_{i}(t)\right] d t-\lambda M .
\end{array}
$$

Here $\kappa_{i}$ is the cost per time unit for observing site $i$. The optimization problem with the relaxed constraint (8) can be expressed as

$$
\underline{\gamma}=\inf _{\pi} \sup _{\lambda} L(\pi, \lambda)=\sup _{\lambda} \inf _{\pi} L(\pi, \lambda)
$$

where the exchange of the supremum and the infimum can be justified using a minimax theorem for constrained dynamic programming [21, chap.1]. Now consider the computation of the dual function

$$
\begin{array}{r}
\underline{\gamma}_{d}(\lambda):=\inf _{\pi} \frac{1}{\varlimsup_{T \rightarrow \infty}} \frac{1}{T} \int_{0}^{T} \sum_{i=1}^{N}\left[\operatorname{Tr}\left(T_{i} \Sigma_{\pi, i}(t)\right)\right. \\
\left.+\left(\kappa_{i}+\lambda\right) \pi_{i}(t)\right] d t-\lambda M,
\end{array}
$$

with $\underline{\gamma}=\sup _{\lambda} \underline{\gamma}_{d}(\lambda)$. The dynamics of the systems are decoupled and the only coupling constraint was adjoined to the objective, so we can compute $\underline{\gamma}_{d}(\lambda)=\sum_{i=1}^{N} \underline{\gamma}^{i}(\lambda)-\lambda M$, where

$\underline{\gamma}^{i}(\lambda):=\inf _{\pi_{i}} \varlimsup_{T \rightarrow \infty} \frac{1}{T} \int_{0}^{T} \operatorname{Tr}\left(T_{i} \Sigma_{\pi_{i}, i}(t)\right)+\left(\kappa_{i}+\lambda\right) \pi_{i}(t) d t$,

by solving individually $N$ similar optimal control problems, one for each system. When the dynamics of the systems are one-dimensional, i.e., $\Sigma_{i} \in \mathbb{R}_{+}$, we can solve the optimal control problem (9) for each site analytically, and hence we obtain an analytical expression of the dual function, which provides a lower bound on the cost for each $\lambda$. Maximizing the concave function $\underline{\gamma}_{d}(\lambda)$ over $\lambda$ yields the performance bound $\underline{\gamma}$, with $\underline{\gamma} \leq \gamma$. The computations are presented in paragraph (III-B). First, we explain how these computations also provide the elements necessary to design a scheduling policy.

\section{A. Restless Bandits}

The RBP was introduced by Whittle in [19] as a generalization of the classical Multi-Armed Bandit Problem (MABP), which was first solved by Gittins [22]. In the RBP, we have $N$ projects evolving independently, $M$ of which can be activated at each time. Projects that are active can evolve according to different dynamics than projects that remain passive. In our problem, the projects correspond to the systems and their activation corresponds to taking a measurement. The active and passive dynamics are described by a Riccati and a Lyapunov differential equation respectively, as explained in the next subsection. We describe the index policy proposed by Whittle for the RBP in our particular context. Although suboptimal in general, Whittle's index policy generalizes the index policy of Gittins', which is known to be optimal in the case of the MABP.

Consider the objective (9) for system $i$. Clearly, the Lagrange multiplier $\lambda$ can be interpreted as a tax penalizing 
measurements of the system. As $\lambda$ increases, the passive action (i.e., not measuring) should become more attractive. For a given value of $\lambda$, let us denote $\mathcal{P}^{i}(\lambda)$ the set of values of $\Sigma_{i}$ (with $\mathcal{P}^{i}(\lambda) \subset \mathbb{R}_{+}$) for which the passive action is optimal. We use the following definition.

Definition 4: System $i$ is indexable if and only if $\mathcal{P}^{i}(\lambda)$ is monotonically increasing (in the sense of set inclusion) from $\emptyset$ to $\mathbb{R}_{+}$as $\lambda$ increases from $-\infty$ to $+\infty$. If system $i$ is indexable, we define its Whittle index as the function $\Sigma_{i} \mapsto$ $\lambda_{i}\left(\Sigma_{i}\right)$, with $\lambda_{i}\left(\Sigma_{i}\right)=\inf \left\{\lambda \in \mathbb{R}: \Sigma_{i} \in \mathcal{P}^{i}(\lambda)\right\}$.

The indexability requirement appears natural, yet Whittle provided an example of an RBP where it is not verified. We will see in the next paragraph, however, that for our particular problem, at least in the scalar case, indexability of the systems is guaranteed. The idea behind the definition of the Whittle index consists in defining an intrinsic "value" for the measurement of system $i$, taking into account both the immediate and future gains. If the covariance of system $i$ is $\Sigma_{i}$, the Whittle index defines this value as the measurement tax (potentially negative) that should be required to make the controller indifferent between measuring and not measuring the system. Finally, if all the systems are indexable, the Whittle policy chooses to measure the $M$ systems with highest current index at each instant. There is significant experimental data and some theoretical evidence indicating that the performance of this policy is often very close to optimal, see e.g. [23]-[25] and section IV-C4.

\section{B. Solution of the Scalar Optimal Control Problem}

We can now consider problem (9) for a single site, dropping the index $i$. In the scalar case, the dynamical evolution of the variance obeys the equation

$$
\dot{\Sigma}=2 A \Sigma+W-\pi \frac{C^{2}}{V} \Sigma^{2}, \quad \text { with } \pi(t) \in\{0,1\} .
$$

The Hamilton-Jacobi-Bellman (HJB) equation is

$$
\begin{array}{r}
\underline{\gamma}(\lambda)=\min \left\{T \Sigma+(2 A \Sigma+W) h^{\prime}(\Sigma ; \lambda),\right. \\
\left.T \Sigma+\kappa+\lambda+\left(2 A \Sigma+W-\frac{C^{2}}{V} \Sigma^{2}\right) h^{\prime}(\Sigma ; \lambda)\right\},
\end{array}
$$

where $h$ is the relative value function. Consider the algebraic Riccati equation (ARE)

$$
2 A x+W-\frac{C^{2}}{V} x^{2}=0 .
$$

First, if $T=0$, it is clearly optimal to always observe if $\lambda+$ $\kappa<0$ and never observe otherwise. Hence the Whittle index is $\lambda(\Sigma)=-\kappa$ for all $\Sigma \in \mathbb{R}_{+}$, and $\gamma(\lambda)=\min \{\kappa+\lambda, 0\}$. So we can now assume $T>0$. If $\bar{C}=0$, again we should always observe if $(\kappa+\lambda)<0$ and never observe otherwise. Hence the Whittle index is again $\lambda(\Sigma)=-\kappa$ for all $\Sigma \in \mathbb{R}_{+}$ and we get

$$
\text { for } \mathrm{C}=0: \underline{\gamma}(\lambda)=\left\{\begin{aligned}
-T & \frac{W}{2 A}+\min \{\kappa+\lambda, 0\} \\
& \text { if the system is stable }(A<0) \\
+\infty & \text { otherwise }(A \geq 0),
\end{aligned}\right.
$$

by letting $\Sigma=-\frac{W}{2 A}$ in the HJB equation for a stable system. The second case is clear from the fact that the system is unstable and cannot be measured. So we can now assume that $C \neq 0$. Then the ARE has two roots

$x_{1}=\frac{A-\sqrt{A^{2}+C^{2} W / V}}{C^{2} / V}, \quad x_{2}=\frac{A+\sqrt{A^{2}+C^{2} W / V}}{C^{2} / V}$.

By assumption $3, W \neq 0$ and so $x_{1}$ is strictly negative and $x_{2}$ is strictly positive. We can treat the case $\kappa+\lambda \leq 0$ immediately. Then it is obviously optimal to always observe, and we get $\underline{\gamma}(\lambda)=T x_{2}+\kappa+\lambda$ by letting $\Sigma=x_{2}$ in the HJB equation.

So from now on we can assume $\lambda>-\kappa$. Let us temporarily assume the following result on the form of the optimal policy. The validity of this assumption can be verified a posteriori from the formulas obtained below, using the fact that the dynamic programming equation provides a sufficient condition for optimality of a solution.

Form of the optimal policy. The optimal policy is a threshold policy, i.e., it observes the system for $\Sigma \geq \Sigma_{t h}$ and does not observe for $\Sigma<\Sigma_{t h}$, for some $\Sigma_{t h} \in \mathbb{R}_{+}$.

We would like to obtain the value of the average cost $\gamma(\lambda)$ and of the threshold $\Sigma_{t h}(\lambda)$. Note that the passive region $\overline{\mathcal{P}}(\lambda)$ of definition 4 is $\mathcal{P}(\lambda)=\left[0, \Sigma_{t h}(\lambda)\right]$, and we already know $\Sigma_{t h}(\lambda)=0$ for $\lambda \leq-\kappa$. The system is indexable if and only if $\Sigma_{t h}(\lambda)$ is an nondecreasing function of $\lambda$, and then inverting the relation $\lambda \mapsto \Sigma_{t h}(\lambda)$ gives the Whittle index $\Sigma \mapsto \lambda(\Sigma)$. The analysis now considers several cases.

1) Case $\Sigma_{t h} \leq x_{2}$ : In this case, we obtain as above

$$
\underline{\gamma}(\lambda)=T x_{2}+\kappa+\lambda,
$$

by letting $\Sigma=x_{2}$ in the HJB equation. Then for $\Sigma \geq \Sigma_{t h}$, we have

$$
\begin{aligned}
T x_{2}+\kappa+\lambda & =T \Sigma+\kappa+\lambda-\frac{C^{2}}{V}\left(\Sigma-x_{2}\right)\left(\Sigma-x_{1}\right) h^{\prime}(\Sigma) \\
\text { so } h^{\prime}(\Sigma) & =\frac{T V}{C^{2}\left(\Sigma-x_{1}\right)},
\end{aligned}
$$

including for the value $\Sigma=x_{2}$ by assuming $h^{\prime}$ to be continuous. By continuity of $h$ at the interface between the active and passive regions, we have

$$
\begin{array}{r}
T \Sigma_{t h}+\left(2 A \Sigma_{t h}+W\right) h^{\prime}\left(\Sigma_{t h}\right)=T \Sigma_{t h}+(\kappa+\lambda) \\
+\left(2 A \Sigma_{t h}+W-\frac{C^{2}}{V} \Sigma_{t h}^{2}\right) h^{\prime}\left(\Sigma_{t h}\right)
\end{array}
$$

i.e.,

$$
\kappa+\lambda=\frac{C^{2}}{V} \Sigma_{t h}^{2} h^{\prime}\left(\Sigma_{t h}\right)=\frac{T \Sigma_{t h}^{2}}{\Sigma_{t h}-x_{1}},
$$

and hence,

$$
\begin{aligned}
\lambda\left(\Sigma_{t h}\right) & =-\kappa+\frac{T \Sigma_{t h}^{2}}{\Sigma_{t h}-x_{1}}, \\
\Sigma_{t h}(\lambda) & =\frac{\frac{\kappa+\lambda}{T}+\sqrt{\left(\frac{\kappa+\lambda}{T}\right)\left(\frac{\kappa+\lambda}{T}-4 x_{1}\right)}}{2} .
\end{aligned}
$$

Expressions (11) and (12), (13) are valid under the condition $\Sigma_{t h}(\lambda) \leq x_{2}$. Note from (12) that $\Sigma_{t h} \mapsto \lambda\left(\Sigma_{t h}\right)$ is an 
increasing function and the functions $\lambda(\cdot)$ and $\Sigma_{t h}(\cdot)$ are inverse of each other.

2) Case $\Sigma_{t h}>x_{2}$ : It turns out that in this case we must distinguish between stable and unstable systems. For a stable system $(A<0)$, the Lyapunov equation

$$
2 A x+W=0
$$

has a strictly positive solution $x_{e}=-\frac{W}{2 A}$, with $x_{e}>x_{2}$ since $C \neq 0$.

Stable System $(A<0)$ with $\Sigma_{t h} \geq x_{e}$ :

In this case we know that $x_{e}$ is in the passive region. Hence, with $\Sigma=x_{e}$ in the HJB equation, we get

$$
\underline{\gamma}(\lambda)=T x_{e}
$$

Then for $\Sigma \leq \Sigma_{t h}$ we have that $T x_{e}=T \Sigma+2 A\left(\Sigma-x_{e}\right) h^{\prime}(\Sigma)$, and so $h^{\prime}(\Sigma)=-\frac{T}{2 A}$, including for $\Sigma=x_{e}$ by assuming $h^{\prime}$ continuous. Again by continuity at the interface between the active and passive regions, we have

$$
\begin{gathered}
\kappa+\lambda=\frac{C^{2}}{V} \Sigma_{t h}^{2} h^{\prime}\left(\Sigma_{t h}\right)=-\frac{C^{2} T}{2 A V} \Sigma_{t h}^{2} \\
\text { so } \lambda\left(\Sigma_{t h}\right)=-\kappa+\frac{C^{2} T \Sigma_{t h}^{2}}{2|A| V}, \quad \Sigma_{t h}(\lambda)=\frac{\sqrt{2|A| V \frac{\lambda+\kappa}{T}}}{|C|} .
\end{gathered}
$$

Stable System $(A<0)$ with $x_{2}<\Sigma_{t h}<x_{e}$, or non-stable system $(A \geq 0)$ :

If the system is marginally stable or unstable, we cannot define $x_{e}$. We can think of this case as $x_{e} \rightarrow \infty$ as $A \rightarrow 0_{-}$, and treat it simultaneously with the case where the system is stable and $x_{2}<\Sigma_{t h}<x_{e}$. Then $x_{2}$ is in the passive region, and $x_{e}$ is in the active region, so the prefactors of $h^{\prime}(x)$ in the HJB equation do not vanish. There is no immediate relation providing the value of $\underline{\gamma}(\lambda)$. We can use the smoothfit principle to handle this case and obtain the expression of the Whittle indices, following [19]. Again the formal justification comes from using the final expression of the value function thus obtained to verify that it indeed satisfies the HJB equation.

Theorem 1 (modification of [19], [26]): Consider a continuous-time one-dimensional restless bandit project $x(t) \in \mathbb{R}$ satisfying

$$
\dot{x}(t)=a_{k}(x), \quad k=0,1,
$$

with passive and active cost rates $r_{k}(x), k=0,1$. Assume that $a_{0}(x)$ does not vanish in the optimal passive region, and $a_{1}(x)$ does not vanish in the optimal active region. Then the Whittle index is given by

$$
\begin{aligned}
\lambda(x)= & r_{0}(x)-r_{1}(x) \\
& +\frac{\left[a_{1}(x)-a_{0}(x)\right]\left[a_{0}(x) r_{1}^{\prime}(x)-a_{1}(x) r_{0}^{\prime}(x)\right]}{a_{0}(x) a_{1}^{\prime}(x)-a_{1}(x) a_{0}^{\prime}(x)} .
\end{aligned}
$$

Remark 5: The assumption that $a_{0}$ and $a_{1}$ do not vanish in the optimal passive and active regions respectively excludes the cases previously studied. It is missing from [19], [26], which therefore provide only an incomplete description of the Whittle indices for one-dimensional continuous-time deterministic projects.
Proof: The derivation of the expression of the Whittle index can be found in [19], [26, p.53], and is valid only under the additional assumption mentioned above.

Corollary 6: The Whittle index for the case $x_{2}<\Sigma_{t h}<x_{e}$ is given by:

$$
\lambda\left(\Sigma_{t h}\right)=-\kappa+\frac{C^{2}}{2 V} \frac{T \Sigma_{t h}^{3}}{A \Sigma_{t h}+W} .
$$

Proof: For $x_{2}<\Sigma_{t h}<x_{e}$, the assumptions of theorem 1 are verified with

$$
\begin{array}{cl}
a_{0}(\Sigma)=2 A \Sigma+W, & a_{1}(\Sigma)=2 A \Sigma+W-\frac{C^{2}}{V} \Sigma^{2} \\
r_{0}(\Sigma)=T \Sigma, & r_{1}(\Sigma)=T \Sigma+\kappa .
\end{array}
$$

The result follows by a straightforward calculation. We can verify that the expression for $\lambda(\Sigma)$ defines an increasing function of $\Sigma$.

With the value of the Whittle index, we can finish the computation of the lower bound $\gamma(\lambda)$ for the case $x_{2}<\Sigma_{t h}<x_{e}$. Inverting the relation (16), we obtain, for a given value of $\lambda$, the boundary $\Sigma_{t h}(\lambda)$ between the passive and active regions. $\Sigma_{t h}(\lambda)$ verifies the depressed cubic equation

$$
X^{3}-\frac{2 V(\lambda+\kappa)}{T C^{2}} A X-\frac{2 V(\lambda+\kappa)}{T C^{2}} W=0 .
$$

For $\lambda+\kappa>0$, by Descartes' rule of signs, this polynomial has exactly one positive root, which is $\Sigma_{t h}(\lambda)$. The HJB equation then reduces to

$$
\begin{array}{r}
\underline{\gamma}(\lambda)=T \Sigma+h^{\prime}(\Sigma)(2 A \Sigma+W), \quad \text { for } \Sigma<\Sigma_{t h}(\lambda) \\
\underline{\gamma}(\lambda)=T \Sigma+\kappa+\lambda+h^{\prime}(\Sigma)\left(2 A \Sigma+W-\frac{C^{2}}{V} \Sigma^{2}\right), \\
\quad \text { for } \Sigma \geq \Sigma_{t h}(\lambda) .
\end{array}
$$

Now for $x_{2}<\Sigma_{t h}(\lambda)<x_{e}$, letting $x=\Sigma_{t h}(\lambda)>0$ in the HJB equation, assuming continuity of $h^{\prime}$ at the boundary of the passive and active regions and eliminating $h^{\prime}\left(\Sigma_{t h}(\lambda)\right)$, we get

$$
\begin{aligned}
\underline{\gamma}(\lambda)= & T \Sigma_{t h}(\lambda)+\kappa+\lambda \\
& +\left(\underline{\gamma}-T \Sigma_{t h}(\lambda)\right)\left(1-\frac{C^{2}}{V} \frac{\left(\Sigma_{t h}(\lambda)\right)^{2}}{2 A \Sigma_{t h}(\lambda)+W}\right),
\end{aligned}
$$

thus,

$$
\left(\underline{\gamma}(\lambda)-T \Sigma_{t h}(\lambda)\right)\left(\frac{C^{2}}{V} \frac{\left(\Sigma_{t h}(\lambda)\right)^{2}}{2 A \Sigma_{t h}(\lambda)+W}\right)=\kappa+\lambda
$$

and hence finally, for $x_{2}<\Sigma_{t h}(\lambda)<x_{e}$ we have

$$
\underline{\gamma}(\lambda)=T \Sigma_{t h}(\lambda)+\frac{V(\kappa+\lambda)\left(2 A \Sigma_{t h}(\lambda)+W\right)}{C^{2}\left(\Sigma_{t h}(\lambda)\right)^{2}} .
$$

3) Summary: We collect the computations above in the following theorem.

Theorem 2: In the one-dimensional Kalman filter scheduling problem with identical sensors, the systems are indexable. For system $i$, the Whittle index $\lambda_{i}\left(\Sigma_{i}\right)$ is given as follows:

- Case $C_{i}=0$ or $T_{i}=0: \lambda_{i}\left(\Sigma_{i}\right)=-\kappa_{i}$, for all $\Sigma_{i} \in \mathbb{R}_{+}$. 
- Case $C_{i} \neq 0$ and $T_{i} \neq 0$ :

$$
\lambda_{i}\left(\Sigma_{i}\right)=\left\{\begin{array}{l}
-\kappa_{i}+\frac{T_{i} \Sigma_{i}^{2}}{\Sigma_{i}-x_{1, i}} \quad \text { if } \Sigma_{i} \leq x_{2, i}, \\
-\kappa_{i}+\frac{C_{i}^{2} T_{i} \Sigma_{i}^{3}}{2 V_{i}\left(A_{i} \Sigma_{i}+W_{i}\right)} \text { if } x_{2, i}<\Sigma_{i}<x_{e, i}, \\
-\kappa_{i}+\frac{T_{i} C_{i}^{2} \Sigma_{i}^{2}}{2\left|A_{i}\right| V_{i}} \text { if } x_{e, i} \leq \Sigma_{i},
\end{array}\right.
$$

with $x_{e, i}$ the root of the Lyapunov equation, the convention $x_{e, i}=+\infty$ if $A_{i} \geq 0$, and $x_{1, i}, x_{2, i}$ the roots of the ARE. The lower bound on the achievable performance is obtained by maximizing the concave function

$$
\underline{\gamma}_{d}(\lambda)=\sum_{i=1}^{N} \underline{\gamma}^{i}(\lambda)-\lambda M
$$

over $\lambda$, where the term $\underline{\gamma}^{i}(\lambda)$ is given by

- Case $T_{i}=0: \underline{\gamma}^{i}(\lambda)=\min \left\{\lambda+\kappa_{i}, 0\right\}$.

- Case $T_{i} \neq 0, \overline{C_{i}}=0: \underline{\gamma}^{i}(\lambda)=\frac{T_{i} W_{i}}{2\left|A_{i}\right|}+\min \left\{\lambda+\kappa_{i}, 0\right\}$ if $A_{i}<0, \gamma^{i}(\lambda)=+\infty$ if $A_{i} \geq 0$.

- Case $C_{i} \neq 0$ and $T_{i} \neq 0$ :

$$
\underline{\gamma}^{i}(\lambda)=\left\{\begin{array}{c}
T_{i} x_{2, i}+\kappa_{i}+\lambda \text { if } \lambda \leq \lambda_{i}\left(x_{2, i}\right), \\
T_{i} \Sigma_{i}^{*}(\lambda)+\frac{V_{i}\left(\kappa_{i}+\lambda\right)\left(2 A_{i} \Sigma_{i}^{*}(\lambda)+W_{i}\right)}{C_{i}^{2}\left(\Sigma_{i}^{*}(\lambda)\right)^{2}} \\
\quad \text { if } \lambda_{i}\left(x_{2, i}\right)<\lambda<\lambda_{i}\left(x_{e, i}\right), \\
T_{i} x_{e, i} \quad \text { if } \lambda_{i}\left(x_{e, i}\right) \leq \lambda .
\end{array}\right.
$$

where in the second case $\Sigma_{i}^{*}(\lambda)$ is the unique positive root of (17).

Proof: The indexability comes from the fact that the indices $\lambda_{i}\left(\Sigma_{i}\right)$ are verified to be monotonically increasing functions of $\Sigma_{i}$. Inverting the relation we obtain $\Sigma_{t h, i}(\lambda)$ as the variance for which we are indifferent between the active and passive actions. As we increase $\lambda, \Sigma_{t h, i}(\lambda)$ increases and the passive region (the interval $\left[0, \Sigma_{t h, i}(\lambda)\right]$ ) increases.

\section{Multidimensional Systems}

Generalizing the computations of the previous section to multidimensional systems requires solving the corresponding optimal control problem in higher dimensions, for which it is not clear that a closed form solution exist. Moreover we have considered in section III a particular case of the sensor scheduling problem where all sensors are identical. We now return to the general multidimensional problem and sensors with possibly distinct characteristics, as described in the introduction.

For the infinite-horizon average cost problem, we show that computing the value of a lower bound similar to the one presented in section III reduces to a convex optimization problem involving, at worst, Linear Matrix Inequalities (LMI) whose size grows polynomially with the problem's essential parameters. Moreover, one can further decompose the computation of this convex program into $N$ coupled subproblems as in the one-dimensional restless bandit case.

\section{A. Performance Bound}

For convenience, let us repeat the deterministic optimal control problem under consideration:

$$
\min _{\pi} \varlimsup_{T \rightarrow \infty} \frac{1}{T} \int_{0}^{T} \sum_{i=1}^{N}\left\{\operatorname{Tr}\left(T_{i} \Sigma_{i}(t)\right)+\sum_{j=1}^{M} \kappa_{i j} \pi_{i j}(t)\right\} d t
$$

s.t. $\dot{\Sigma}_{i}(t)=A_{i} \Sigma_{i}+\Sigma_{i} A_{i}^{T}+W_{i}$

$$
-\Sigma_{i}\left(\sum_{j=1}^{M} \pi_{i j}(t) C_{i j}^{T} V_{i j}^{-1} C_{i j}\right) \Sigma_{i}, i=1 \ldots, N,
$$

$\pi_{i j}(t) \in\{0,1\}, \forall t \geq 0, i=1 \ldots, N, j=1, \ldots, M$,

$\sum_{i=1}^{N} \pi_{i j}(t) \leq 1, \forall t \geq 0, \quad j=1, \ldots, M$,

$\sum_{j=1}^{M} \pi_{i j}(t) \leq 1, \forall t \geq 0, \quad i=1, \ldots, N$,

$\Sigma_{i}(0)=\Sigma_{i, 0}, i=1, \ldots, N$.

Here we consider the constraints (1) and (3), but any combination of inequality and equality constraints from (1)-(4) can be used without changing the argument for the derivation of the performance bound. We define the following quantities:

$$
\tilde{\pi}_{i j}(T)=\frac{1}{T} \int_{0}^{T} \pi_{i j}(t) d t, \forall T \geq 0 .
$$

Since $\pi_{i j}(t) \in\{0,1\}$ we must have $0 \leq \tilde{\pi}_{i j}(T) \leq 1$. Our first goal, inspired by the idea already exploited in the restless bandit problem, is to obtain a lower bound on the cost of the finite-horizon optimal control problem in terms of the numbers $\tilde{\pi}_{i j}(T)$ instead of the functions $\pi_{i j}(t)$.

It will be easier to work with the information matrices $Q_{i}(t)=\Sigma_{i}^{-1}(t)$. Hence we replace the dynamics (22) by the equivalent

$$
\begin{gathered}
\dot{Q}_{i}=-Q_{i} A_{i}-A_{i}^{T} Q_{i}-Q_{i} W_{i} Q_{i}+\sum_{j=1}^{M} \pi_{i j}(t) C_{i j}^{T} V_{i j}^{-1} C_{i j} \\
i=1, \ldots, N
\end{gathered}
$$

Let us also define, for all $T \geq 0$,

$$
\tilde{\Sigma}_{i}(T):=\frac{1}{T} \int_{0}^{T} \Sigma_{i}(t) d t, \quad \tilde{Q}_{i}(T):=\frac{1}{T} \int_{0}^{T} Q_{i}(t) d t .
$$

By linearity of the trace operator, we can rewrite the objective function

$$
\varlimsup_{T \rightarrow \infty} \sum_{i=1}^{N}\left\{\operatorname{Tr}\left(T_{i} \tilde{\Sigma}_{i}(T)\right)+\sum_{j=1}^{M} \kappa_{i j} \tilde{\pi}_{i j}(T)\right\} .
$$

Let $\mathbb{S}^{n}, \mathbb{S}_{+}^{n}, \mathbb{S}_{++}^{n}$ denote the set of symmetric, symmetric positive semidefinite and symmetric positive definite matrices respectively. A function $f: \mathbb{R}^{m} \rightarrow \mathbb{S}^{n}$ is called matrix convex if and only if for all $x, y \in \mathbb{R}^{m}$ and $\alpha \in[0,1]$, we have

$$
f(\alpha x+(1-\alpha) y) \preceq \alpha f(x)+(1-\alpha) f(y),
$$

where $\preceq$ refers to the usual partial order on $\mathbb{S}^{n}$, i.e., $A \preceq B$ if and only if $B-A \in \mathbb{S}_{+}^{n}$. Equivalently, $f$ is matrix convex if 
the scalar functions $x \mapsto z^{T} f(x) z$ are convex for all vectors $z$. The following lemma will be useful

Lemma 7: The functions

$$
\begin{array}{lr}
\mathbb{S}_{++}^{n} \rightarrow \mathbb{S}_{++}^{n} & \mathbb{S}^{n} \rightarrow \mathbb{S}^{n} \\
X \mapsto X^{-1} & X \mapsto X W X
\end{array}
$$

for $W \in \mathbb{S}_{+}^{n}$, are matrix convex.

Proof: See [27, p.76, p.110].

A consequence of this lemma is that Jensen's inequality is valid for these functions. We use it first as follows

$$
\forall T, \quad\left(\frac{1}{T} \int_{0}^{T} \Sigma_{i}(t) d t\right)^{-1} \preceq \frac{1}{T} \int_{0}^{T} Q_{i}(t) d t=\tilde{Q}_{i}(T),
$$

hence

$$
\forall T, \quad \tilde{\Sigma}_{i}(T) \succeq\left(\tilde{Q}_{i}(T)\right)^{-1}
$$

and so

$$
\operatorname{Tr}\left(T_{i} \tilde{\Sigma}_{i}(T)\right) \geq \operatorname{Tr}\left(T_{i}\left(\tilde{Q}_{i}(T)\right)^{-1}\right) .
$$

Next, integrating (26) and letting $Q_{i, 0}=\Sigma_{i, 0}^{-1}$, we have

$\frac{1}{T}\left(Q_{i}(T)-Q_{i, 0}\right)=-\tilde{Q}_{i}(T) A_{i}-A_{i}^{T} \tilde{Q}_{i}(T)$

$-\frac{1}{T} \int_{0}^{T} Q_{i}(t) W_{i} Q_{i}(t) d t+\sum_{j=1}^{M}\left(\frac{1}{T} \int_{0}^{T} \pi_{i j}(t) d t\right) C_{i j}^{T} V_{i j}^{-1} C_{i j}$,

i.e.,

$$
\begin{aligned}
& \frac{1}{T}\left(Q_{i}(T)-Q_{i, 0}\right)=-\tilde{Q}_{i}(T) A_{i}-A_{i}^{T} \tilde{Q}_{i}(T) \\
& -\frac{1}{T} \int_{0}^{T} Q_{i}(t) W_{i} Q_{i}(t) d t+\sum_{j=1}^{M} \tilde{\pi}_{i j}(T) C_{i j}^{T} V_{i j}^{-1} C_{i j} .
\end{aligned}
$$

Using Jensen's inequality and lemma 7 again, we have

$$
\frac{1}{T} \int_{0}^{T} Q_{i}(t) W_{i} Q_{i}(t) d t \succeq \tilde{Q}_{i}(T) W_{i} \tilde{Q}_{i}(T),
$$

and so we obtain

$$
\begin{aligned}
\frac{1}{T}\left(Q_{i}(T)\right. & \left.-Q_{i, 0}\right) \preceq-\tilde{Q}_{i}(T) A_{i}-A_{i}^{T} \tilde{Q}_{i}(T) \\
& -\tilde{Q}_{i}(T) W_{i} \tilde{Q}_{i}(T)+\sum_{j=1}^{M} \tilde{\pi}_{i j}(T) C_{i j}^{T} V_{i j}^{-1} C_{i j} .
\end{aligned}
$$

Last, since $Q_{i}(T) \succeq 0$, this implies, for all $T$,

$$
\begin{aligned}
\tilde{Q}_{i}(T) A_{i}+A_{i}^{T} \tilde{Q}_{i}(T)+\tilde{Q}_{i}(T) W_{i} \tilde{Q}_{i}(T) & \\
& -\sum_{j=1}^{M} \tilde{\pi}_{i j}(T) C_{i j}^{T} V_{i j}^{-1} C_{i j} \preceq \frac{Q_{i, 0}}{T} .
\end{aligned}
$$

So we see that for a fixed policy $\pi$ and any time $T$, the quantity

$$
\sum_{i=1}^{N}\left\{\operatorname{Tr}\left(T_{i} \tilde{\Sigma}_{i}(T)\right)+\sum_{j=1}^{M} \kappa_{i j} \tilde{\pi}_{i j}(T)\right\}
$$

is bounded below by the quantity

$$
\sum_{i=1}^{N}\left\{\operatorname{Tr}\left(T_{i}\left(\tilde{Q}_{i}(T)\right)^{-1}\right)+\sum_{j=1}^{M} \kappa_{i j} \tilde{\pi}_{i j}(T)\right\}
$$

where the matrices $\tilde{Q}_{i}(T)$ and the number $\tilde{\pi}_{i j}(T)$ are subject to the constraints $(28)$ as well as

$$
\begin{aligned}
& 0 \leq \tilde{\pi}_{i j}(T) \leq 1, i=1, \ldots, N, j=1, \ldots, M, \\
& \sum_{i=1}^{N} \tilde{\pi}_{i j}(T) \leq 1, j=1, \ldots, M, \\
& \sum_{j=1}^{M} \tilde{\pi}_{i j}(T) \leq 1, i=1, \ldots, N .
\end{aligned}
$$

Hence for any $T$, the quantity $Z^{*}(T)$ defined below is a lower bound on the value of (29) for any choice of policy $\pi$

$$
\begin{aligned}
& Z^{*}(T)=\min _{Q_{i}, p_{i j}} \sum_{i=1}^{N}\left\{\operatorname{Tr}\left(T_{i} Q_{i}^{-1}\right)+\sum_{j=1}^{M} \kappa_{i j} p_{i j}\right\}, \quad \\
& \text { s.t. } Q_{i} A_{i}+A_{i}^{T} Q_{i}+Q_{i} W_{i} Q_{i}-\sum_{j=1}^{M} p_{i j} C_{i j}^{T} V_{i j}^{-1} C_{i j} \preceq \frac{Q_{i, 0}}{T}, \\
& Q_{i} \succ 0, i=1 \ldots, N, \\
& 0 \leq p_{i j} \leq 1, i=1 \ldots, N, j=1, \ldots, M, \\
& \sum_{i=1}^{N} p_{i j} \leq 1, j=1, \ldots, M, \sum_{j=1}^{M} p_{i j} \leq 1, i=1, \ldots, N .
\end{aligned}
$$

Now replace the right-hand side of (31) by zero, to get

$$
Q_{i} A_{i}+A_{i}^{T} Q_{i}+Q_{i} W_{i} Q_{i}-\sum_{j=1}^{M} p_{i j} C_{i j}^{T} V_{i j}^{-1} C_{i j} \preceq 0,
$$

for $i=1 \ldots, N$, and call the corresponding optimum value $Z^{*}$. Defining $\delta:=1 / T$, and rewriting with a slight abuse of notation $Z^{*}(\delta)$ instead of $Z^{*}(T)$ for $\delta$ positive, we also define $Z^{*}(0)=Z^{*}$. Note that $Z^{*}(0)$ is finite, since we can find a feasible solution as follows. For each $i$, form a matrix $P=\left[p_{i j}\right]$ satisfying the constraints and such that $p_{i j}>0$ for all $i, j$. Such a matrix is easy to find if we consider the inequality constraints (1) and (3), since we can simply take $p_{i j}=1 / \max (M, N)$ for all $i, j$. If equality constraints are involved instead, such a matrix $P$ exists as a consequence of Birkhoff theorem [28], see Theorem 8. Now we consider the quadratic inequality (32) for some value of $i$. From the detectability assumption 2 and the choice of $p_{i j}$, we deduce that the pair $\left(A_{i}, \hat{C}_{i}\right)$, with

$$
\hat{C}_{i}=\left[\begin{array}{lll}
\sqrt{p_{i 1}} C_{i 1}^{T} V_{i 1}^{-1 / 2} & \cdots & \sqrt{p_{i M}} C_{i M}^{T} V_{i M}^{-1 / 2}
\end{array}\right]^{T}
$$

is detectable. Also note that

$$
\hat{C}_{i}^{T} \hat{C}_{i}=\sum_{j=1}^{M} p_{i j} C_{i j}^{T} V_{i j}^{-1} C_{i j} .
$$

Together with the controllability assumption 3 , we then know that (32) has a positive definite solution $\tilde{Q}_{i}[29$, theorem 2.4.25]. Hence $Z^{*}(0)$ is finite.

We can also define $Z^{*}(\delta)$ for $\delta<0$, by changing the righthand side of (31) into $\delta Q_{i, 0}=-|\delta| Q_{i, 0}$. We have that $Z^{*}(\delta)$ is finite for $\delta<0$ and $|\delta|$ small enough. Indeed, passing the term $\delta Q_{i, 0}$ on the left hand side, this can then be seen as a 
perturbation of the matrix $\hat{C}_{i}$ above, and for $|\delta|$ small enough, detectability, which is an open condition, is preserved. Now we will see below that (30) is a convex program. It is then a standard result of perturbation analysis (see e.g. [27, p. 250]) that $Z^{*}(\delta)$ is a convex function of $\delta$, hence continuous on the interior of its domain, in particular continuous at $\delta=0$. So

$$
\varlimsup_{T \rightarrow \infty} Z^{*}(T)=\lim _{T \rightarrow \infty} Z^{*}(T)=Z^{*} .
$$

Finally, for any policy $\pi$, we obtain the following lower bound on the achievable cost

$$
\begin{aligned}
\varlimsup_{T \rightarrow \infty} \frac{1}{T} \int_{0}^{T} \sum_{i=1}^{N}\left\{\operatorname{Tr}\left(T_{i} \Sigma_{i}(t)\right)\right. & \left.+\sum_{j=1}^{M} \kappa_{i j} \pi_{i j}(t)\right\} d t \\
& \geq \lim _{T \rightarrow \infty} Z^{*}(T)=Z^{*} .
\end{aligned}
$$

We now show how to compute $Z^{*}$ by solving a convex program involving linear matrix inequalities. For each $i$, introduce a new (slack) matrix variable $R_{i}$. Since $Q_{i} \succ 0, R_{i} \succeq Q_{i}^{-1}$ is equivalent, by taking the Schur complement, to

$$
\left[\begin{array}{cc}
R_{i} & I \\
I & Q_{i}
\end{array}\right] \succeq 0
$$

and the Riccati inequality (32) can be rewritten

$$
\left[\begin{array}{cc}
Q_{i} A_{i}+A_{i}^{T} Q_{i}-\sum_{j=1}^{M} p_{i j} C_{i j}^{T} V_{i j}^{-1} C_{i j} & Q_{i} W_{i}^{1 / 2} \\
W_{i}^{1 / 2} Q_{i} & -I
\end{array}\right] \preceq 0 .
$$

We finally obtain the semidefinite program

$$
\begin{aligned}
& Z^{*}=\min _{R_{i}, Q_{i}, p_{i j}} \sum_{i=1}^{N}\left\{\operatorname{Tr}\left(T_{i} R_{i}\right)+\sum_{j=1}^{M} \kappa_{i j} p_{i j}\right\} \\
& \text { s.t. }\left[\begin{array}{cc}
R_{i} & I \\
I & Q_{i}
\end{array}\right] \succeq 0, Q_{i} \succ 0, i=1, \ldots, N, \\
& {\left[\begin{array}{cc}
Q_{i} A_{i}+A_{i}^{T} Q_{i}-\sum_{j=1}^{M} p_{i j} C_{i j}^{T} V_{i j}^{-1} C_{i j} & Q_{i} W_{i}^{1 / 2} \\
W_{i}^{1 / 2} Q_{i} & -I
\end{array}\right] \preceq 0,} \\
& 0 \leq p_{i j} \leq 1, i=1 \ldots, N, j=1, \ldots, M, \\
& \sum_{i=1}^{N} p_{i j} \leq 1, j=1, \ldots, M, \\
& \sum_{j=1}^{M} p_{i j} \leq 1, i=1, \ldots, N .
\end{aligned}
$$

Hence solving the program (34) provides a lower bound on the achievable cost for the original optimal control problem.

\section{B. Problem Decomposition}

It is well-know that efficient methods exist to solve (34) in polynomial time, which implies a computation time polynomial in the number of variables of the original problem. Still, as the number of targets increases, the large semidefinite program (34) becomes difficult to solve. Note however that it can be decomposed into $N$ small coupled programs, following the dual decomposition approach already used for the restless bandit problem. This decomposition is sometimes useful to solve large scale programs with a large number of systems. For completeness, we present the argument in more details below.

We first note that (35) is the only constraint that links the $N$ subproblems together. So we form the Lagrangian

$$
\begin{array}{r}
L(R, Q, p ; \lambda)=\sum_{i=1}^{N}\left\{\operatorname{Tr}\left(T_{i} R_{i}\right)+\sum_{j=1}^{M}\left(\kappa_{i j}+\lambda_{j}\right) p_{i j}\right\} \\
-\sum_{j=1}^{M} \lambda_{j},
\end{array}
$$

where $\lambda \in \mathbb{R}_{+}^{M}$ is a vector of Lagrange multipliers. We would take $\lambda \in \mathbb{R}^{M}$ if we had the constraint (2) instead of (1). Now the dual function is

$$
G(\lambda)=\sum_{i=1}^{N} G_{i}(\lambda)-\sum_{j=1}^{M} \lambda_{j},
$$

with, for each $i=1, \ldots, N$,

$$
\begin{aligned}
& G_{i}(\lambda)=\min _{R_{i}, Q_{i},\left\{p_{i j}\right\}_{j=1}^{M}} \operatorname{Tr}\left(T_{i} R_{i}\right)+\sum_{j=1}^{M}\left(\kappa_{i j}+\lambda_{j}\right) p_{i j}, \\
& \text { s.t. }\left[\begin{array}{cc}
R_{i} & I \\
I & Q_{i}
\end{array}\right] \succeq 0, Q_{i} \succ 0, \\
& {\left[\begin{array}{cc}
Q_{i} A_{i}+A_{i}^{T} Q_{i}-\sum_{j=1}^{M} p_{i j} C_{i j}^{T} V_{i j}^{-1} C_{i j} & Q_{i} W_{i}^{1 / 2} \\
W_{i}^{1 / 2} Q_{i} & -I
\end{array}\right] \preceq 0,} \\
& \sum_{j=1}^{M} p_{i j} \leq 1, \quad 0 \leq p_{i j} \leq 1, j=1, \ldots, M .
\end{aligned}
$$

Note that each program (37) involves only two matrix variables in contrast to (34), which has $2 N$ matrix variables.

The optimization algorithm proceeds then as follows [30, chap. 11]. We choose an initial value $\lambda^{1} \geq 0$ and set $k=1$.

1) For $i=1, \ldots, N$, compute $R_{i}^{k}, Q_{i}^{k},\left\{p_{i j}^{k}\right\}_{1 \leq j \leq M}$ optimal solution of (37), and the value $G_{i}\left(\lambda^{k}\right)$.

2) The value of the dual function at $\lambda^{k}$ is given by (36). A supergradient of $G\left(\lambda^{k}\right)$ at $\lambda^{k}$ is given by

$$
\left[\sum_{i=1}^{N} p_{i 1}^{k}-1, \ldots, \sum_{i=1}^{N} p_{i M}^{k}-1\right] .
$$

3) Compute $\lambda^{k+1}$ in order to maximize $G(\lambda)$. We can do this by using a supergradient algorithm, or any preferred nonsmooth optimization algorithm. Increment $k$ and go to step 1, or stop according to some convergence criterion.

Because the initial program (34) is convex and strictly feasible, we know that the optimal value of the dual optimization problem is equal to the optimal value of the primal. Moreover, the optimal variables of the primal are obtained at step 1 of the algorithm above once convergence has been reached. 


\section{Open-loop Periodic Policies Achieving the Performance Bound}

1) Definition of the Policies: In this section we describe a sequence of open-loop policies that can approach the lower bound computed by (34) arbitrarily closely, thus proving that this bound is tight. These policies are periodic switching strategies using a schedule obtained from the optimal parameters $p_{i j}$. Assuming no switching times or costs, their performance approaches the bound as the length of the switching cycle decreases toward 0 .

Let $P=\left[p_{i j}\right]_{1 \leq i \leq N, 1 \leq j \leq M}$ be the matrix of optimal parameters obtained in the solution of (34). We assume here that constraints (1) and (3) are enforced, which is the most general case for the discussion in this section. Hence $P$ verifies

$0 \leq p_{i j} \leq 1, i=1, \ldots, N, j=1, \ldots, M$,

$\sum_{i=1}^{N} p_{i j} \leq 1, j=1, \ldots, M, \quad$ and $\sum_{j=1}^{M} p_{i j} \leq 1, i=1, \ldots, N$.

A doubly substochastic matrix of dimension $n$ is an $n \times n$ matrix $A=\left[a_{i j}\right]_{1 \leq i, j \leq n}$ which satisfies

$$
\begin{aligned}
& 0 \leq a_{i j} \leq 1, i, j=1, \ldots, n, \\
& \sum_{i=1}^{n} a_{i j} \leq 1, j=1, \ldots, n, \quad \text { and } \sum_{j=1}^{n} a_{i j} \leq 1, i=1, \ldots, n .
\end{aligned}
$$

If $M=N, P$ is therefore a doubly substochastic matrix. Else if $M<N$ (resp. $N<M$ ) we can add $N-M$ columns of zeros (resp. $M-N$ rows of zeros) to $P$ to obtain a doubly substochastic matrix. In any case, we call the resulting doubly substochastic matrix $\tilde{P}=\left[\tilde{p}_{i j}\right]$. If rows are added, this is equivalent to the initial problem with additional "dummy systems". If columns are added, they correspond to using "dummy sensors". Dummy systems (i.e., for $i>N$ ) are not included in the objective function (the corresponding $T_{i}$ is 0 ), and a dummy sensor (i.e., for $j>M$ ) is associated formally to the matrices $V_{i j}^{-1}=0$ for all $i$, in effect producing no measurement. In the following we assume that $\tilde{P}$ is an $N \times N$ doubly substochastic matrix, but the discussion in the $M \times M$ case is identical. Doubly substochastic matrices have been intensively studied, and the material used in the following can be found in the book of Marshall and Olkin [31]. In particular, we have the following corollary of a classical theorem of Birkhoff [28], which says that a doubly stochastic matrix is a convex combination of permutation matrices.

Theorem 8 ( [32]): The set of $N \times N$ doubly substochastic matrices is the convex hull of the set $\mathcal{P}_{0}$ of $N \times N$ matrices which have a most one unit in each row and each column, and all other entries are zero.

Hence, for the doubly substochastic matrix $\tilde{P}$, there exists a set of positive numbers $\phi_{k}$ and matrices $P_{k} \in \mathcal{P}_{0}$ such that

$$
\tilde{P}=\sum_{k=1}^{K} \phi_{k} P_{k}, \text { with } \sum_{k=1}^{K} \phi_{k}=1, \text { for some integer } K \text {. }
$$

One way of computing this decomposition is to first extend $\tilde{P}$ to the $2 N \times 2 N$ doubly stochastic matrix

$$
\hat{P}=\left[\begin{array}{cc}
\tilde{P} & I-D_{r} \\
I-D_{c} & \tilde{P}^{T}
\end{array}\right],
$$

where $r_{1}, \ldots, r_{N}$ and $c_{1}, \ldots, c_{N}$ are the row sums and column sums of $\tilde{P}$, and $D_{r}=\operatorname{diag}\left(r_{1}, \ldots, r_{N}\right), D_{c}=$ $\operatorname{diag}\left(c_{1}, \ldots, c_{N}\right)$. Then there is an algorithm that runs in time $O\left(N^{4.5}\right)$ [31], [33] and provides the decomposition

$$
\hat{P}=\sum_{k=1}^{K} \phi_{k} \hat{P}_{k}
$$

with $K \leq(2 N-1)^{2}+1$ and where the $\hat{P}_{k}$ 's are permutation matrices of size $2 N \times 2 N$. The decomposition (38) is finally obtained by deleting the last $N$ rows and columns of $\hat{P}_{k}$ to obtain the matrices $P_{k}, k=1, \ldots, K$.

Note that any matrix $A=\left[a_{i j}\right]_{i, j} \in \mathcal{P}_{0}$ represents a valid sensor/system assignment (for the problem with additional dummy systems or sensors), where sensor $j$ is measuring system $i$ if and only if $a_{i j}=1$. With the decomposition (38), we now consider a family of periodic switching policies parametrized by a positive number $\epsilon$ representing a time interval over which the switching schedule is executed completely. For a given value of $\epsilon$, the policy is defined as follows:

1) At time $t=l \epsilon, l \in \mathbb{N}$, associate sensor $j$ to system $i$ as specified by the matrix $P_{1}$ of the representation (38). Run the corresponding continuous-time Kalman filters, keeping this sensor/system association for a duration $\phi_{1} \epsilon$.

2) At time $t=\left(l+\phi_{1}\right) \epsilon$, switch to the assignment specified by $P_{2}$. Run the corresponding continuous time Kalman filters until $t=\left(l+\phi_{1}+\phi_{2}\right) \epsilon$.

3) Repeat the switching procedure, switching to matrix $P_{i+1}$ at time $t=l+\phi_{1}+\cdots+\phi_{i}$, for $i=1, \ldots, K-1$.

4) At time $t=\left(l+\phi_{1}+\cdots+\phi_{K}\right) \epsilon=(l+1) \epsilon$, start the switching sequence again at step 1 with $P_{1}$ and repeat the steps above.

It is easy to see that the matrices $P_{i}, i=1, \ldots, K$ never specify that a "dummy sensor" should execute a measurement or that a "dummy system" should be measured, since from the decomposition (38) this would correspond to nonzero entries in the columns or rows added to $P$ to form $\tilde{P}$.

2) Performance of the Periodic Switching Policies: Let us fix $\epsilon>0$ in the definition of the switching policy, and consider now, for this policy, the evolution of the covariance matrix $\Sigma_{i}^{\epsilon}(t)$ for the estimation error on the state of system $i$. The superscript indicates the dependence of the policy on the period $\epsilon$. First we have

Lemma 9: For all $i \in\{1, \ldots, N\}$, the estimation error covariance $\Sigma_{i}^{\epsilon}(t)$ converges as $t \rightarrow \infty$ to a periodic function $\bar{\Sigma}_{i}^{\epsilon}(t)$ of period $\epsilon$.

Proof: Fix $i \in\{1, \ldots, N\}$. Let $\sigma_{i}(t) \in\{0,1, \ldots, N\}$ be the function specifying which sensor is observing system $i$ at time $t$ under the switching policy. By convention $\sigma_{i}(t)=0$ means that no sensor is scheduled to observe system $i$, and $\sigma_{i}(t)=j$ means that sensor $j$ measures system $i$. Note from the remark following the description of the switching policies 
that in fact we have $\sigma_{i}(t) \in\{0, \ldots, M\}$, i.e., the policy never schedules measurements by dummy sensors. Similarly, if instead we were considering the situation $M>N$ and $\tilde{P}$ an $M \times M$ matrix, then we would have $\sigma_{i}(t)=0$ for $i \in\{N+1, \ldots, M\}$ and all $t$. Note also that $\sigma_{i}(t)$ is a piecewise constant, $\epsilon$-periodic function. The switching times of $\sigma_{i}(t)$ are $t=\left(l+\phi_{1}+\cdots+\phi_{k-1}\right) \epsilon$, for $k=1, \ldots, K$ and $l \in \mathbb{N}$.

The covariance matrix $\Sigma_{i}^{\epsilon}(t)$ obeys the following periodic Riccati differential equation (PRE):

$$
\begin{aligned}
\dot{\Sigma}_{i}^{\epsilon}(t)= & A_{i} \Sigma_{i}^{\epsilon}(t)+\Sigma_{i}^{\epsilon}(t) A_{i}^{T}+W_{i} \\
& -\Sigma_{i}^{\epsilon}(t)\left(C_{i}^{\epsilon}(t)\right)^{T} C_{i}^{\epsilon}(t) \Sigma_{i}^{\epsilon}(t) \\
\Sigma_{i}^{\epsilon}(t)= & \Sigma_{i, 0},
\end{aligned}
$$

where

$$
C_{i}^{\epsilon}(t):=V_{i \sigma_{i}(t)}^{-1 / 2} C_{i \sigma_{i}(t)}
$$

is a piecewise constant, $\epsilon$-periodic matrix valued function, and we use the convention $V_{i j}^{-1}=C_{i j}=0$ when $j=0$. We now show that $\left(A_{i}, C_{i}^{\epsilon}(\cdot)\right)$ is detectable. Let $j_{1}, \ldots, j_{K}$ be the successive values taken by the function $\sigma_{i}(t)$ over the period $\epsilon$. From the definition of detectability for linear periodic systems and it modal characterization [34, p.130], we immediately deduce that the pair $\left(A_{i}, C_{i}^{\epsilon}(\cdot)\right)$ is not detectable if and only if there exists an eigenpair $(\lambda, x)$ for $A_{i}$, with $\operatorname{Re}(\lambda) \geq 0$, $x \neq 0$, such that

$$
A_{i} x=\lambda x, \text { and } C_{i}^{\epsilon}(t) e^{A_{i} t} x=e^{\lambda t} C_{i}^{\epsilon}(t) x=0, \forall t \in[0, \epsilon],
$$

hence,

$$
C_{i j_{1}} x=\ldots=C_{i j_{K}} x=0 .
$$

Let us denote by $p_{k, i j}$ the $(i, j)^{\text {th }}$ element of the matrix $P_{k}$ in the decomposition (38). We have $p_{k, i j}=1_{\left\{j=j_{k}\right\}}$ with the above definition of $j_{k}$, including the case $j_{k}=0$ (no measurement), which gives $p_{k, i j}=0$ for all $j \in\{1, \ldots, N\}$. Then we can write

$$
\begin{aligned}
& \sum_{k=1}^{K} \phi_{k} C_{i j_{k}}^{T} V_{i j_{k}}^{-1} C_{i j_{k}}=\sum_{k=1}^{K} \phi_{k}\left(\sum_{j=1}^{N} p_{k, i j} C_{i j}^{T} V_{i j}^{-1} C_{i j}\right) \\
& =\sum_{j=1}^{N}\left(\sum_{k=1}^{K} \phi_{k} p_{k, i j}\right) C_{i j}^{T} V_{i j}^{-1} C_{i j}=\sum_{j=1}^{N} \tilde{p}_{i j} C_{i j}^{T} V_{i j}^{-1} C_{i j} \\
& =\sum_{j=1}^{M} p_{i j} C_{i j}^{T} V_{i j}^{-1} C_{i j}=\hat{C}_{i}^{T} \hat{C}_{i}
\end{aligned}
$$

where the next-to-last equality uses the fact that $\tilde{p}_{i j}=p_{i j}$ for $j \leq M$ and $\tilde{p}_{i j}=0$ for $j \geq M+1$, and $\hat{C}_{i}$ was defined in (33). Note that we now consider this definition of $\hat{C}_{i}$ for the optimal parameters $p_{i j}$ provided by the solution of (34). Then (41) and (42) imply $\left\|\hat{C}_{i} x\right\|_{2}=0$, so $\hat{C}_{i} x=0$, i.e., $\left(A_{i}, \hat{C}_{i}\right)$ is not detectable. But the parameters $p_{i j}$ being optimal for the program (34), this would imply that this program is not feasible [29, p.68], a contradiction with our discussion following (33). So $\left(A_{i}, C_{i}^{\epsilon}(\cdot)\right)$ must be detectable.
This result, together with our assumption 1 and the corollary to Theorem 3 in [35, p. 95], yields

$$
\lim _{t \rightarrow \infty}\left(\Sigma_{i}^{\epsilon}(t)-\bar{\Sigma}_{i}^{\epsilon}(t)\right)=0
$$

where $\bar{\Sigma}_{i}^{\epsilon}(t)$ is the strong solution of the PRE, which is $\epsilon$ periodic.

Next, denote by $\tilde{\Sigma}_{i}(t)$ the solution to the following Riccati differential equation (RDE)

$$
\begin{aligned}
& \dot{\Sigma}_{i}=A_{i} \Sigma_{i}+\Sigma_{i} A_{i}^{T}+W_{i}-\Sigma_{i}\left(\sum_{j=1}^{M} p_{i j} C_{i j}^{T} V_{i j}^{-1} C_{i j}\right) \Sigma_{i}, \\
& \Sigma_{i}(0)=\Sigma_{i, 0} .
\end{aligned}
$$

Assumptions 2 and 3, together with our discussion of the implied detectability of the pair $\left(A, \hat{C}_{i}\right)$ (see (42)), guarantee that $\tilde{\Sigma}_{i}(t)$ converges to a positive definite limit denoted $\Sigma_{i}^{*}$. Moreover, $\Sigma_{i}^{*}$ is stabilizing and is the unique positive definite solution to the algebraic Riccati equation (ARE):

$$
A_{i} \Sigma_{i}+\Sigma_{i} A_{i}^{T}+W_{i}-\Sigma_{i}\left(\sum_{j=1}^{M} p_{i j} C_{i j}^{T} V_{i j}^{-1} C_{i j}\right) \Sigma_{i}=0
$$

The next lemma says that the periodic function $\bar{\Sigma}_{i}^{\epsilon}(t)$ oscillates in a neighborhood of $\Sigma_{i}^{*}$.

Lemma 10: For all $t \in \mathbb{R}_{+}$, we have $\bar{\Sigma}_{i}^{\epsilon}(t)-\Sigma_{i}^{*}=O(\epsilon)$ as $\epsilon \rightarrow 0$.

Proof: The function $t \rightarrow \bar{\Sigma}_{i}^{\epsilon}(t)$ of lemma 9 is the strong periodic solution of the PRE (39). It is $\epsilon$-periodic and positive semidefinite. From Radon's lemma [29, p.90], which gives a representation of the solution to a Riccati differential equation as the ratio of solutions to a linear ODE, we also know that $\bar{\Sigma}_{i}^{\epsilon}$ is $C^{\infty}$ on each interval where $\sigma_{i}(t)$ is constant, where $\sigma_{i}(t)$ is the switching signal defined in the proof of Lemma 9.

Let $\hat{\Sigma}_{i}^{\epsilon}$ be the average of $t \rightarrow \bar{\Sigma}_{i}^{\epsilon}(t)$ :

$$
\hat{\Sigma}_{i}^{\epsilon}=\frac{1}{\epsilon} \int_{0}^{\epsilon} \bar{\Sigma}_{i}^{\epsilon}(t) d t
$$

and note that $\hat{\Sigma}_{i}^{\epsilon}$ is positive semidefinite. From the preceding remarks, it is easy to deduce that we have $\bar{\Sigma}_{i}^{\epsilon}(t)-\hat{\Sigma}_{i}^{\epsilon}=O(\epsilon)$ for all $t$. Then, averaging the PRE (39) over the interval $[0, \epsilon]$, we obtain

$$
\begin{aligned}
& A_{i} \hat{\Sigma}_{i}^{\epsilon}+\hat{\Sigma}_{i}^{\epsilon} A^{T}+W_{i}-\frac{1}{\epsilon} \int_{0}^{\epsilon} \bar{\Sigma}_{i}^{\epsilon}(t)\left(C_{i}^{\epsilon}(t)\right)^{T} C_{i}^{\epsilon}(t) \bar{\Sigma}_{i}^{\epsilon}(t) d t \\
& =\frac{1}{\epsilon}\left(\bar{\Sigma}_{i}^{\epsilon}(\epsilon)-\bar{\Sigma}_{i}^{\epsilon}(0)\right)=0,
\end{aligned}
$$

where $C_{i}^{\epsilon}(t)$ was defined in (40). Expanding this equation in powers of $\epsilon$, we get

$$
\begin{aligned}
& A_{i} \hat{\Sigma}_{i}^{\epsilon}+\hat{\Sigma}_{i}^{\epsilon} A^{T}+W_{i} \\
& -\hat{\Sigma}_{i}^{\epsilon}\left(\frac{1}{\epsilon} \int_{0}^{\epsilon}\left(C_{i}^{\epsilon}(t)\right)^{T} C_{i}^{\epsilon}(t) d t\right) \hat{\Sigma}_{i}^{\epsilon}+R(\epsilon)=0,
\end{aligned}
$$


where $R(\epsilon)=O(\epsilon)$. Let $j_{k}:=\sigma_{i}(t)$ for $t \in\left[\left(l+\phi_{1}+\ldots+\right.\right.$ $\left.\left.\phi_{k-1}\right) \epsilon,\left(l+\phi_{1}+\ldots+\phi_{k}\right) \epsilon\right]$. We can then rewrite, using (42),

$$
\begin{aligned}
\frac{1}{\epsilon} \int_{0}^{\epsilon}\left(C_{i}^{\epsilon}(t)\right)^{T} C_{i}^{\epsilon}(t) d t & =\sum_{k=1}^{K} \phi_{k} C_{i j_{k}}^{T} V_{i j_{k}}^{-1} C_{i j_{k}} \\
& =\sum_{j=1}^{M} p_{i j} C_{i j}^{T} V_{i j}^{-1} C_{i j} .
\end{aligned}
$$

So we obtain

$$
\begin{aligned}
& A_{i} \hat{\Sigma}_{i}^{\epsilon}+\hat{\Sigma}_{i}^{\epsilon} A^{T}+\left(W_{i}+R(\epsilon)\right) \\
& -\hat{\Sigma}_{i}^{\epsilon}\left(\sum_{j=1}^{M} p_{i j} C_{i j}^{T} V_{i j}^{-1} C_{i j}\right) \hat{\Sigma}_{i}^{\epsilon}=0 .
\end{aligned}
$$

Note moreover that for $\epsilon$ sufficiently small, $\hat{\Sigma}_{i}^{\epsilon}$ is the unique positive semidefinite stabilizing solution of this ARE, using the fact that controllability of $\left(A_{i}, W_{i}^{1 / 2}\right)$ is an open condition. Now comparing this ARE to the ARE (44), and since the stabilizing solution of an ARE is a real analytic function of the parameters [36], we deduce that $\hat{\Sigma}_{i}^{\epsilon}-\Sigma_{i}^{*}=O(\epsilon)$, and the lemma.

Theorem 11: Let $Z^{\epsilon}$ denote the performance of the periodic switching policy with period $\epsilon$. Then $Z^{\epsilon}-Z^{*}=O(\epsilon)$ as $\epsilon \rightarrow 0$, where $Z^{*}$ is the performance bound (34). Hence the performance of the switching policy approaches the lower bound arbitrarily closely as the period tends to 0 .

Proof: We have

$$
Z^{\epsilon}=\varlimsup_{T \rightarrow \infty} \frac{1}{T} \int_{0}^{T} \sum_{i=1}^{N}\left(\operatorname{Tr}\left(T_{i} \Sigma_{i}^{\epsilon}(t)\right)+\sum_{j=1}^{M} \kappa_{i j} \pi_{i j}^{\epsilon}(t)\right) d t,
$$

where $\pi^{\epsilon}$ is the sensor/system assignment of the switching policy. First by using a transformation similar to (42) and using the convention $\kappa_{i j}=0$ for $j \in\{0\} \cup\{M+1, \ldots, N\}$ (no measurement or measurement by a dummy sensor), we have for system $i$

$$
\begin{aligned}
& \frac{1}{T} \int_{0}^{T} \sum_{j=1}^{M} \kappa_{i j} \pi_{i j}^{\epsilon}(t) d t= \frac{1}{T} \sum_{n=0}^{\left\lfloor\frac{T}{\epsilon}\right\rfloor-1} \int_{n \epsilon}^{(n+1) \epsilon} \sum_{j=1}^{M} \kappa_{i j} \pi_{i j}^{\epsilon}(t) d t \\
&+\frac{1}{T} \int_{\left\lfloor\frac{T}{\epsilon}\right\rfloor \epsilon}^{T} \sum_{j=1}^{M} \kappa_{i j} \pi_{i j}^{\epsilon}(t) d t \\
&= \frac{1}{T}\left\lfloor\frac{T}{\epsilon}\right\rfloor \sum_{k=1}^{K} \kappa_{i j_{k}}\left(\phi_{k} \epsilon\right)+\frac{1}{T} \int_{\left\lfloor\frac{T}{\epsilon}\right\rfloor \epsilon}^{T} \sum_{j=1}^{M} \kappa_{i j} \pi_{i j}^{\epsilon}(t) d t \\
&=\left\lfloor\frac{T}{\epsilon}\right\rfloor \frac{\epsilon}{T} \sum_{j=1}^{M} \kappa_{i j} p_{i j}+\frac{1}{T} \int_{\left\lfloor\frac{T}{\epsilon}\right\rfloor \epsilon}^{T} \sum_{j=1}^{M} \kappa_{i j} \pi_{i j}^{\epsilon}(t) d t
\end{aligned}
$$

where the $j_{k}$ 's were defined in the proof of Lemma 9. Hence

$$
\lim _{T \rightarrow \infty} \frac{1}{T} \int_{0}^{T} \sum_{i=1}^{N} \sum_{j=1}^{M} \kappa_{i j} \pi_{i j}^{\epsilon}(t) d t=\sum_{i=1}^{N} \sum_{j=1}^{M} \kappa_{i j} p_{i j} .
$$

Next, from Lemmas 9 and 10, it follows readily that $\varlimsup_{t \rightarrow \infty} \Sigma_{i}^{\epsilon}(t)-\Sigma_{i}^{*}=O(\epsilon)$. Since $\Sigma_{i}^{*} \succ 0$, we can define $Q_{i}^{*}=\left(\Sigma_{i}^{*}\right)^{-1}$. Then under our assumptions $Q_{i}^{*}$ is a maximal (for the partial order on $\mathbb{S}_{+}^{n}$ ) solution of the quadratic matrix inequality

$$
Q_{i} A_{i}+A_{i}^{T} Q_{i}+Q_{i} W_{i} Q_{i}-\sum_{j=1}^{M} p_{i j} C_{i j}^{T} V_{i j}^{-1} C_{i j} \preceq 0,
$$

see e.g. [37, cor. 13.13]. Hence for the $p_{i j}$ obtained from the computation of the lower bound (34), these matrices $Q_{i}^{*}$ minimize the function $\sum_{i=1}^{N} \operatorname{Tr}\left(T_{i} Q_{i}^{-1}\right)$ over the matrices $Q_{i}$ satisfying (46). Thus we obtain a minimizer for (30), with constraints (32). In conclusion, the covariance matrices resulting from the switching policies approach within $O(\epsilon)$ as $\epsilon \rightarrow 0$ the covariance matrices that are obtained from the lower bound on the achievable cost. The theorem follows, by bounding above the upper limit of a sum by the sum of the upper limits to get $Z^{*} \leq Z^{\epsilon} \leq Z^{*}+O(\epsilon)$.

Remark 12: Since the bound computed in (34) is tight, and since it is easy to see that the performance bound of section III is at least as good as the bound (34) for the simplified problem of that section, we conclude that the two bounds coincide and that section III gives an alternative way of computing the solution of (34) in the case of identical sensors and onedimensional systems. Using the closed form expression for the dual function (20), we only need in that case to optimize over the single Lagrange multiplier $\lambda$, independently of the number $N$ of systems, instead of solving the LMI (34), whose number of variables grows with $N$.

3) Transient Behavior of the Switching Policies: Before concluding, we take a look at the transient behavior of the switching policies. We show that over a finite time interval, $\Sigma_{i}^{\epsilon}(t)$ remains close to $\tilde{\Sigma}_{i}(t)$, solution of the "averaged" RDE (43). Together with the previous result of Lemma 9 on the asymptotic behavior, we see then that $\Sigma_{i}^{\epsilon}(t)$ and $\tilde{\Sigma}_{i}(t)$ remain close for all $t$. For a matrix $A$, we denote by $\|A\|_{\infty}$ the maximal absolute value of the entries of $A$.

Lemma 13: For all $0 \leq T_{0}<\infty$, there exist constants $\epsilon_{0}>$ 0 and $M_{0}>0$ such that for all $0<\epsilon \leq \epsilon_{0}$ and for all $t \in\left[0, T_{0}\right]$, we have $\left\|\Sigma_{i}^{\epsilon}(t)-\tilde{\Sigma}_{i}(t)\right\|_{\infty} \leq M_{0} \epsilon$.

Proof: As in the proof of lemma 10, by Radon's lemma we know that $\Sigma_{i}^{\epsilon}$ is $C^{\infty}$ on each interval where $\sigma_{i}(t)$ is constant. We have then, over the interval $t \in\left[l \epsilon,\left(l+\phi_{1}\right) \epsilon\right]$, for $l \in \mathbb{N}$ :

$$
\begin{aligned}
\Sigma_{i}^{\epsilon}\left(\left(l+\phi_{1}\right) \epsilon\right)= & \Sigma_{i}^{\epsilon}(l \epsilon)+\phi_{1} \epsilon\left[A_{i} \Sigma_{i}^{\epsilon}(l \epsilon)+\Sigma_{i}^{\epsilon}(l \epsilon) A_{i}^{T}+W_{i}\right. \\
& \left.-\Sigma_{i}^{\epsilon}(l \epsilon) C_{i j_{1}}^{T} V_{i j_{1}}^{-1} C_{i j_{1}} \Sigma_{i}^{\epsilon}(l \epsilon)\right]+O\left(\epsilon^{2}\right),
\end{aligned}
$$

where as before we denote $j_{k}:=\sigma_{i}(t)$ for $t \in\left[\left(l+\phi_{1}+\right.\right.$ $\left.\left.\ldots+\phi_{k-1}\right) \epsilon,\left(l+\phi_{1}+\ldots+\phi_{k}\right) \epsilon\right]$. Now over the period $t \in$ $\left[\left(l+\phi_{1}\right) \epsilon,\left(l+\phi_{1}+\phi_{2}\right) \epsilon\right]$, we have:

$\Sigma_{i}^{\epsilon}\left(\left(l+\phi_{1}+\phi_{2}\right) \epsilon\right)=$ $\Sigma_{i}^{\epsilon}\left(\left(l+\phi_{1}\right) \epsilon\right)+\phi_{2} \epsilon\left[A_{i} \Sigma_{i}^{\epsilon}\left(\left(l+\phi_{1}\right) \epsilon\right)+\Sigma_{i}^{\epsilon}\left(\left(l+\phi_{1}\right) \epsilon\right) A_{i}^{T}\right.$

$\left.+W_{i}-\Sigma_{i}^{\epsilon}\left(\left(l+\phi_{1}\right) \epsilon\right) C_{i j_{2}}^{T} V_{i j_{2}}^{-1} C_{i j_{2}} \Sigma_{i}^{\epsilon}\left(\left(l+\phi_{1}\right) \epsilon\right)\right]+O\left(\epsilon^{2}\right)$.

Using (47), we deduce that

$$
\begin{aligned}
& \Sigma_{i}^{\epsilon}\left(\left(l+\phi_{1}+\phi_{2}\right) \epsilon\right)= \\
& \Sigma_{i}^{\epsilon}(l \epsilon)+\epsilon\left[\phi_{1}+\phi_{2}\right]\left\{A_{i} \Sigma_{i}^{\epsilon}(l \epsilon)+\Sigma_{i}^{\epsilon}(l \epsilon) A_{i}^{T}+W_{i}\right\} \\
& -\epsilon \Sigma_{i}^{\epsilon}(l \epsilon)\left(\phi_{1} C_{i j_{1}}^{T} V_{i j_{1}}^{-1} C_{i j_{1}}+\phi_{2} C_{i j_{2}}^{T} V_{i j_{2}}^{-1} C_{i j_{2}}\right) \Sigma_{i}^{\epsilon}(l \epsilon)+O\left(\epsilon^{2}\right) .
\end{aligned}
$$


By immediate induction, and since $\phi_{1}+\cdots+\phi_{K}=1$, we then have

$$
\begin{array}{r}
\Sigma_{i}^{\epsilon}((l+1) \epsilon)=\Sigma_{i}^{\epsilon}(l \epsilon)+\epsilon\left\{A_{i} \Sigma_{i}^{\epsilon}(l \epsilon)+\Sigma_{i}^{\epsilon}(l \epsilon) A_{i}^{T}+W_{i}\right. \\
\left.-\Sigma_{i}^{\epsilon}(l \epsilon)\left(\sum_{k=1}^{K} \phi_{k} C_{i j_{k}}^{T} V_{i j_{k}}^{-1} C_{i j_{k}}\right) \Sigma_{i}^{\epsilon}(l \epsilon)\right\}+O\left(\epsilon^{2}\right) .
\end{array}
$$

Hence by (42), $\Sigma_{i}^{\epsilon}$ verifies the relation

$$
\begin{aligned}
& \Sigma_{i}^{\epsilon}((l+1) \epsilon)=\Sigma_{i}^{\epsilon}(l \epsilon)+\epsilon\left\{A_{i} \Sigma_{i}^{\epsilon}(l \epsilon)+\Sigma_{i}^{\epsilon}(l \epsilon) A_{i}^{T}+W_{i}\right. \\
& \left.-\Sigma_{i}^{\epsilon}(l \epsilon)\left(\sum_{j=1}^{M} p_{i j} C_{i j}^{T} V_{i j}^{-1} C_{i j}\right) \Sigma_{i}^{\epsilon}(l \epsilon)\right\}+O\left(\epsilon^{2}\right) .
\end{aligned}
$$

But notice now that the approximation (48) is also true by definition for $\tilde{\Sigma}_{i}(t)$ over the interval $t \in[l \epsilon,(l+1) \epsilon]$. Next, consider the following identity for $Q, X$ and $\tilde{X}$ symmetric matrices:

$$
\begin{aligned}
& A \tilde{X}+\tilde{X} A^{T}-\tilde{X} Q \tilde{X}-\left(A X+X A^{T}-X Q X\right) \\
& =(A-\tilde{X} Q)(\tilde{X}-X)+(\tilde{X}-X)(A-\tilde{X} Q)^{T} \\
& \quad+(\tilde{X}-X) Q(\tilde{X}-X) .
\end{aligned}
$$

Letting $Q=\sum_{j=1}^{M} p_{i j} C_{i j}^{T} V_{i j}^{-1} C_{i j}, \Delta_{i}^{\epsilon}(l)=\tilde{\Sigma}_{i}(l \epsilon)-\Sigma_{i}^{\epsilon}(l \epsilon)$, we obtain from this identity

$$
\begin{aligned}
& \Delta_{i}^{\epsilon}(l+1)=\Delta_{i}^{\epsilon}(l)+\epsilon\left\{\left(A-\tilde{\Sigma}_{i}(l \epsilon) Q\right) \Delta_{i}^{\epsilon}(l)\right. \\
& \left.\quad+\Delta_{i}^{\epsilon}(l)\left(A-\tilde{\Sigma}_{i}(l \epsilon) Q\right)^{T}+\Delta_{i}^{\epsilon}(l) Q \Delta_{i}^{\epsilon}(l)\right\}+O\left(\epsilon^{2}\right) .
\end{aligned}
$$

Note that $\Delta_{i}^{\epsilon}(0)=0$ and $\tilde{\Sigma}_{i}(t)$ is bounded, so by immediate induction we have

$$
\Delta_{i}^{\epsilon}(l)=\sum_{k=1}^{l} R_{k}(\epsilon), \quad \text { where } R_{k}(\epsilon)=O\left(\epsilon^{2}\right) \text { for all } k .
$$

Fix $T_{0} \geq 0$. We have then

$$
\tilde{\Sigma}_{i}\left(\left\lceil\frac{T_{0}}{\epsilon}\right\rceil \epsilon\right)-\Sigma_{i}^{\epsilon}\left(\left\lceil\frac{T_{0}}{\epsilon}\right\rceil \epsilon\right)=\Delta_{i}^{\epsilon}\left(\left\lceil\frac{T_{0}}{\epsilon}\right\rceil\right)=O(\epsilon) .
$$

This means that there exist constants $\epsilon_{0}, M_{0}>0$ such that

$$
\left\|\tilde{\Sigma}_{i}\left(\left\lceil\frac{T_{0}}{\epsilon}\right\rceil \epsilon\right)-\Sigma_{i}^{\epsilon}\left(\left\lceil\frac{T_{0}}{\epsilon}\right\rceil \epsilon\right)\right\|_{\infty} \leq M_{0} \epsilon,
$$

for all $0<\epsilon<\epsilon_{0}$. It is easy to see from the argument above that a similar approximation is in fact valid for all $t$ up to time $\left\lceil\frac{T_{0}}{\epsilon}\right\rceil \epsilon$.

4) Numerical Simulation: On Fig. 1, we compare the covariance trajectories for Whittle's index policy, the periodic switching policy and the greedy policy (measuring the system with highest current mean square error on the estimate) for a simple problem with one sensor switching between two scalar systems. Significant improvements over the greedy policy can be obtained in general by using the periodic switching policies or the Whittle policy. An important computational advantage of the Whittle policy for large-scale problems with a limited number of identical sensors is that by using the closed form solution of the indices provided in section III-B3, it requires only ordering $N$ numbers (which is the same computational

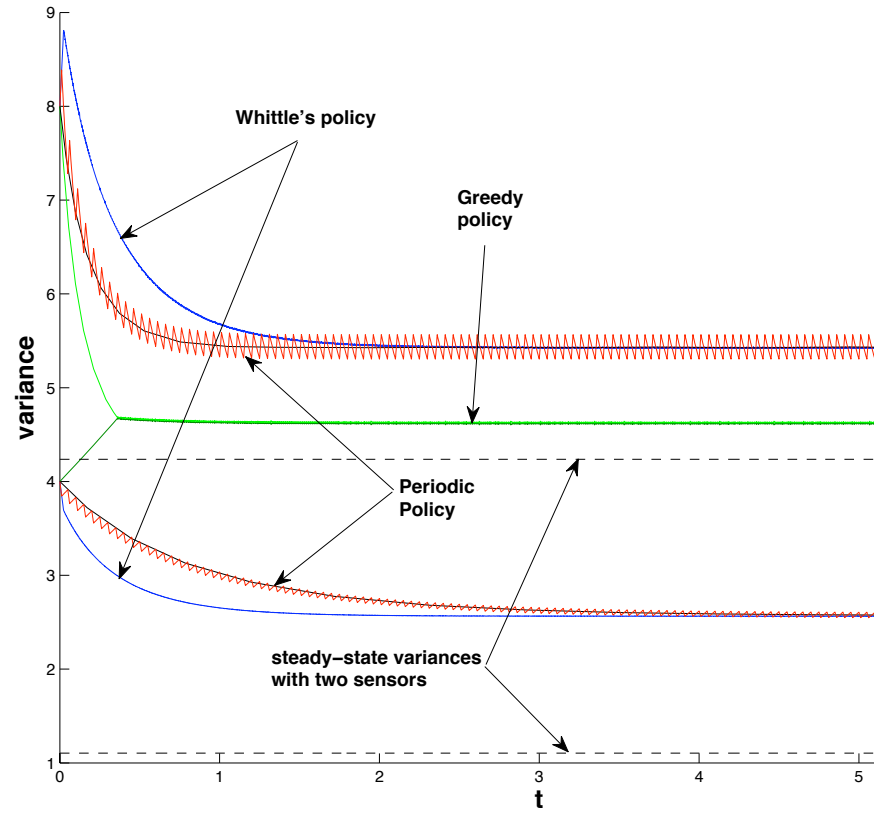

Fig. 1. Comparison of the variance trajectories under the periodic switching policy, Whittle's index policy, and the greedy policy. For the last two policies, we assumed that instantaneous switching was possible. Also shown are the trajectories of $\tilde{\Sigma}_{i}(t)$, solution of the RDE (43) (curves averaging the oscillations). Here a single sensor switches between two scalar systems. The period $\epsilon$ was chosen to be 0.05 . The system parameters are $A_{1}=0.1, A_{2}=$ $2, C_{i}=W_{i}=V_{i}=1, \kappa_{i}=0$. The dashed lines are the steady-state values that could be achieved with two identical sensors, each measuring one system. The performance of the Whittle policy is 7.99, which is optimal (i.e., matches the bound). The performance of the greedy policy is 9.2 . Note that the greedy policy makes the variances converge, while the Whittle policy makes the Whittle indices (not shown) converge. The switching policy spends $22.9 \%$ of its time measuring system 1 and $77.1 \%$ of its time measuring system 2 .

cost as for the greedy policy), whereas designing the openloop switching policy requires computing the solution of the program (34). On Fig. 2, we plot the performance of the periodic switching policy for the same example, as the period $\epsilon$ increases. It is interesting to note the graceful performance degradation, which seems to extend the intuition of Theorem 11 to relatively large period lengths.

Finally, we show an example of simulations for a largerscale problem. We consider the scheduling of 30 sensors measuring the state of a single 20-dimensional system. The problem parameters (matrices and costs) are randomly generated. This results in an LMI with 450 variables, which can be solved in a few seconds on a standard laptop using CVXOPT [38]. The subsequent Birkhoff-von Neumann decomposition only takes a few milliseconds. To illustrate a quick extension of the basic LMI (34), we added the linear constraint $\sum_{j=1}^{30} p_{1 j} \leq \rho$ with $\rho \in(0,1]$. This constraint models the requirement that only a fraction $\rho$ of each period can be spent measuring the system, due for example to the controller implementation platform executing other tasks. We call the time where no sensor measures the system the "passive mode", and plot the performance bound provided by the LMI as a function of $1-\rho$ on Fig. 3. The cost blows up as $\rho$ approaches 0 because the system turned out to be unstable in this example. 


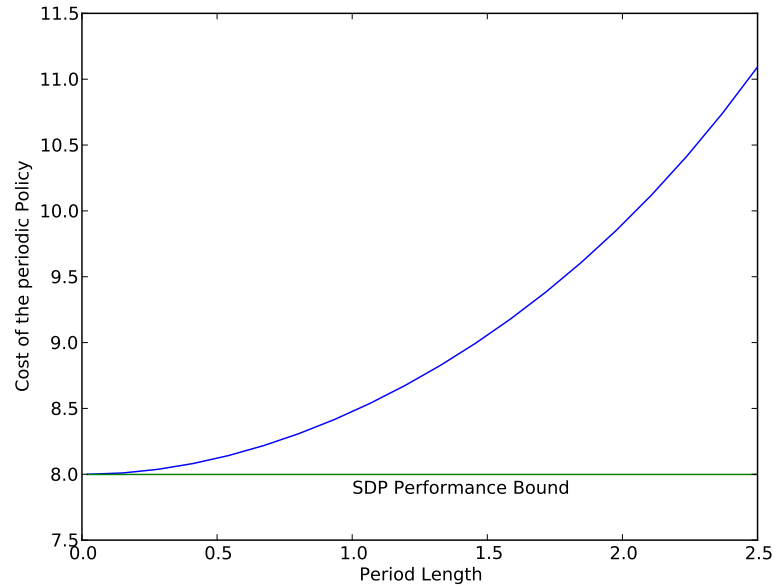

Fig. 2. For the system of Fig. 1, we compute the cost of the periodic policy as the duration $\epsilon$ of each schedule increases, from 0.02 to 2.5 .

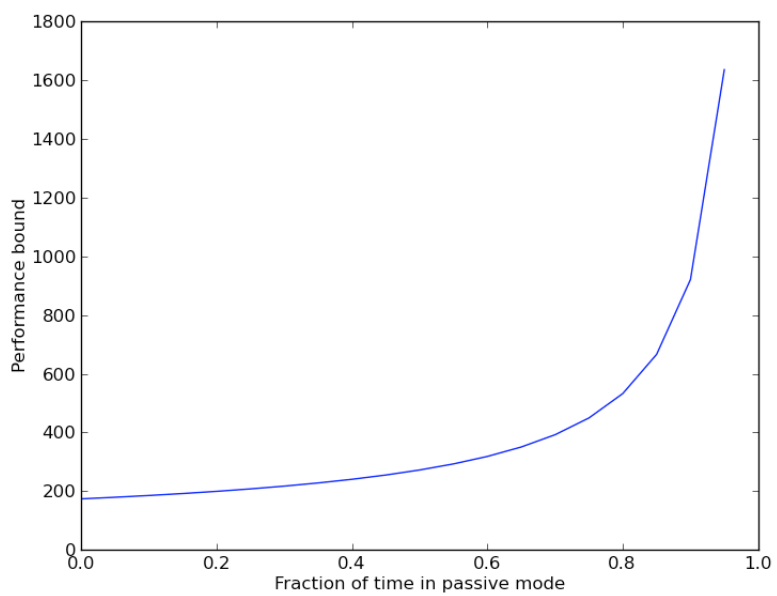

Fig. 3. Computation of the performance bound for a random problem with a 20-dimensional plant and 30 sensors, as a function of the time in each scheduling period where no measurement can be made (passive mode).

\section{CONCLUSiON}

In this paper, we have considered an attention-control problem in continuous time, which consists of scheduling sensor/target assignments and running the corresponding Kalman filters. We proved that the bound obtained from a relaxation inspired by the RBP is tight, assuming we allow the sensors to switch arbitrarily fast between the targets. An open question is to characterize the performance of the restless bandit index policy derived in the scalar case. It was found experimentally that the performance of this policy seems to match the bound, but we do not have a proof of this fact. Obtaining optimal policies in a feedback form for the multidimensional case would also be of interest. For practical applications, the main limitation of our model concerns the absence of switching costs and delays. Still, the optimal solution obtained in the absence of such costs should provide insight into the derivation of heuristics for more complex models. Additionally there are numerous sensor scheduling applications, such as for telemetry-data aerospace systems or radar waveform selection systems, where the switching costs are not too important.

\section{REFERENCES}

[1] R. Evans, V. Krishnamurthy, G. Nair, and L. Sciacca, "Networked sensor management and data rate control for tracking maneuvering targets," IEEE Transactions on Signal Processing, vol. 53, no. 6, pp. 1979-1991, June 2005.

[2] V. Gupta, T. H. Chung, B. Hassibi, and R. M. Murray, "On a stochastic sensor selection algorithm with applications in sensor scheduling and sensor coverage," Automatica, vol. 42, no. 2, pp. 251-260, 2006.

[3] L. Zhang and D. Hristu-Varsakelis, "Communication and control codesign for networked control systems," Automatica, vol. 42, no. 953-958, 2006.

[4] J. Williams, "Information theoretic sensor management," Ph.D. dissertation, Massachusetts Institute of Technology, February 2007.

[5] L. Meier, J. Perschon, and R. Dressler, "Optimal control of measurement systems," IEEE Transactions on Automatic Control, vol. 12, no. 5, pp. 528-536, 1967.

[6] A. Tiwari, "Geometrical analysis of spatio-temporal planning problems," Ph.D. dissertation, California Institute of Technology, 2006.

[7] M. Athans, "On the determination of optimal costly measurement strategies for linear stochastic systems," Automatica, vol. 8, pp. 397412, 1972.

[8] E. Skafidas and A. Nerode, "Optimal measurement scheduling in linear quadratic Gaussian control problems," in Proceedings of the IEEE International Conference on Control Applications, Trieste, Italy, 1998, pp. 1225-1229.

[9] A. Savkin, R. Evans, and E. Skafidas, "The problem of optimal robust sensor scheduling," Systems and Control Letters, vol. 43, pp. 149-157, 2001.

[10] J. Baras and A. Bensoussan, "Optimal sensor scheduling in nonlinear filtering of diffusion processes," SIAM Journal on Control and Optimization, vol. 27, no. 4, pp. 786-813, 1989.

[11] H. Lee, K. Teo, and A. Lim, "Sensor scheduling in continuous time," Automatica, vol. 37, pp. 2017-2023, 2001.

[12] E. Feron and C. Olivier, "Targets, sensors and infinite-horizon tracking optimality," in Proceedings of the 29th IEEE Conference on Decision and Control, 1990.

[13] Y. Oshman, "Optimal sensor selection strategy for discrete-time state estimators," IEEE Transactions on Aerospace and Electronic Systems, vol. 30, no. 2, pp. 307-314, 1994.

[14] A. Tiwari, M. Jun, D. E. Jeffcoat, and R. M. Murray, "Analysis of dynamic sensor coverage problem using Kalman filters for estimation," in Proceedings of the 16th IFAC World Congress, 2005.

[15] B. F. La Scala and B. Moran, "Optimal target tracking with restless bandits,” Digital Signal Processing, vol. 16, pp. 479-487, 2006.

[16] L. Shi, M. Epstein, B. Sinopoli, and R. M. Murray, "Effective sensor scheduling schemes in a sensor network by employing feedback in the communication loop," in Proceedings of the 16th IEEE International Conference on Control Applications, 2007.

[17] A. I. Mourikis and S. I. Roumeliotis, "Optimal sensor scheduling for resource-constrained localization of mobile robot formations," IEEE Transactions on Robotics, vol. 22, no. 5, pp. 917-931, 2006.

[18] K. J. Åström and B. Wittenmark, Computer-Controller Systems: Theory and Design. Prentice Hall, 1997.

[19] P. Whittle, "Restless bandits: activity allocation in a changing world," Journal of Applied Probability, vol. 25A, pp. 287-298, 1988.

[20] R. E. Kalman and R. S. Bucy, "New results in linear filtering and prediction," Journal of Basic Engineering (ASME), vol. 83D, pp. 95$108,1961$.

[21] E. Altman, Constrained Markov Decision Processes. Chapman and Hall, 1999.

[22] J. Gittins and D. Jones, "A dynamic allocation index for the sequential design of experiments," in Progress in Statistics, J. Gani, Ed. Amsterdam: North-Holland, 1974, pp. 241-266.

[23] K. Glazebrook, D. Ruiz-Hernandez, and C. Kirkbride, "Some indexable families of restless bandit problems," Advances in Applied Probability, vol. 38, pp. 643-672, 2006.

[24] R. Weber and G. Weiss, "On an index policy for restless bandits." Journal of Applied Probability, vol. 27, pp. 637-648, 1990. 
[25] J. Le Ny, M. Dahleh, and E. Feron, "Multi-UAV dynamic routing with partial observations using restless bandit allocation indices," in Proceedings of the 2008 American Control Conference, Seattle, June 2008.

[26] F. Dusonchet, "Dynamic scheduling for production systems operating in a random environment," Ph.D. dissertation, Ecole Polytechnique Fédérale de Lausanne, 2003.

[27] S. P. Boyd and L. Vandenberghe, Convex Optimization. Cambridge University Press, 2006.

[28] G. Birkhoff, "Tres observaciones sobre el algebra lineal," Univ. Nac. Tucuman Rev. Ser. A, vol. 5, pp. 147-151, 1946.

[29] H. Abou-Kandil, G. Freiling, V. Ionescu, and G. Jank, Matrix Riccati Equations in Control and Systems Theory, ser. Systems \& Control: Foundations \& Applications. Birkhäuser, 2003.

[30] J. F. Bonnans, J. C. Gilbert, C. Lemaréchal, and C. A. Sagastizábal, Numerical Optimization: Theoretical and Practical Aspects. Springer, 2006.

[31] A. Marshall and I. Olkin, Inequalities: theory of majorization and its applications. Academic Press, 1979.

[32] L. Mirsky, "On a convex set of matrices," Archiv der Mathematik, vol. 10, pp. 88-92, 1959.

[33] C. Chang, W. Chen, and H. Huang, "Birkhoff-von Neumann input buffered crossbar switches," INFOCOM 2000. Nineteenth Annual Joint Conference of the IEEE Computer and Communications Societies. Proceedings. IEEE, vol. 3, 2000.

[34] S. Bittanti, P. Colaneri, and G. D. Nicolao, "The periodic Riccati equation," in The Riccati equation, S. Bittanti, A. J. Laub, and J. C. Willems, Eds. Springer-Verlag, 1991.

[35] G. De Nicolao, "On the convergence to the strong solution of periodic Riccati equations," International Journal of Control, vol. 56, no. 1, pp. 87-97, 1992.

[36] D. Delchamps, "Analytic feedback control and the algebraic Riccati equation," IEEE Transactions on Automatic Control, vol. 29, no. 11, pp. 1031-1033, 1984.

[37] K. Zhou, J. C. Doyle, and K. Glover, Robust and Optimal Control. Prentice Hall, 1996.

[38] J. Dahl and L. Vandenberghe, "CVXOPT: A Python package for convex optimization," 2008, http://abel.ee.ucla.edu/cvxopt/.

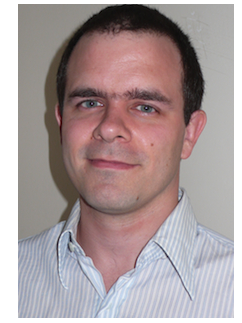

Jerome Le Ny (S'05 - M'09) received the Bachelor's degree from the Ecole Polytechnique, France, in 2001, the M.Sc. degree in Electrical Engineering from the University of Michigan, Ann Arbor, in 2003, and the Ph.D. degree in Aeronautics and Astronautics from MIT in 2008. From December 2003 to August 2004, he was a software engineer with Robert Bosch GmbH, Saint-Ouen, France. He is currently a Postdoctoral Researcher with the GRASP Laboratory at the University of Pennsylvania. His research interests include robust and stochastic control with applications to autonomous and embedded systems, air traffic control, and the associated computational methods supporting the design of complex systems.

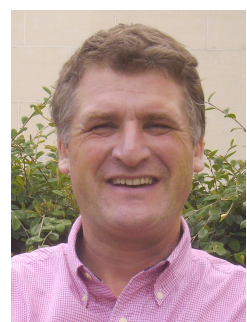

Eric Feron (M'94) is the Dutton-Ducoffe professor of aerospace software engineering at the Georgia Institute of Technology. A former student of Ecole Polytechnique, France, he received his M.S. in computer science from Ecole Polytechnique and Ecole Normale Supérieure, France, and his Ph.D. in aeronautics and astronautics from Stanford University, in 1990 and 1994, respectively. Following an engineering appointment with the ministry of defense, France, he was on the faculty of the Massachusetts Institute of Technology in the department of aeronautics and astronautics from 1993 to 2005. His research interests are the application of computer science, control and optimization theories to important aerospace problems, including flight control systems and air transportation. $\mathrm{He}$ is a co-author of the monograph Linear Matrix Inequalities in System and Control Theory (SIAM, 1994), and the English translation of Bézout's "General Theory of Algebraic Equations" (Princeton, 2006).

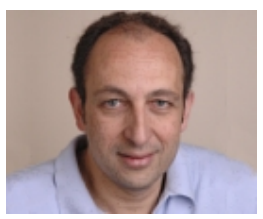

Munther A. Dahleh (S'84 - M'87 - SM'97 - F'01) was born in 1962. He received the B.S. degree from Texas A \& M university, College Station, Texas in 1983, and his Ph.D. degree from Rice University, Houston, TX, in 1987, all in Electrical Engineering. Since then, he has been with the Department of Electrical Engineering and Computer Science, MIT, Cambridge, MA, where he is now a full Professor. $\mathrm{He}$ is currently the associate director of the Laboratory for Information and Decision Systems. He has been a visiting Professor at the Department of Electrical Engineering, California Institute of Technology, Pasadena, CA, for the Spring of 1993. He has held consulting positions with several companies in the US and abroad.

Dr. Dahleh has been the recipient of the Ralph Budd award in 1987 for the best thesis at Rice University, George Axelby outstanding paper award (paper coauthored with J.B. Pearson in 1987), an NSF presidential young investigator award (1991), the Finmeccanica career development chair (1992) and the Donald P. Eckman award from the American Control Council in 1993, the Graduate Students Council teaching award in 1995, the George Axelby outstanding paper award (paper coauthored with Bamieh and Paganini in 2004), and the Hugo Schuck Award for Theory (for the paper coauthored with Martins). He became a fellow of IEEE in year 2000. He was a plenary speaker at the 1994 American Control Conference, at the Mediterranean Conference on Control and Automation in 2003, at the MTNS in 2006, at SYSID in 2009, and at Asian Control Conference in 2009. He was an Associate Editor for IEEE Transactions On Automatic Control and for Systems and Control Letters. He is the co-author (with Ignacio Diaz-Bobillo) of the book Control of Uncertain Systems: A Linear Programming Approach, published by PrenticeHall, and the co-author (with Nicola Elia) of the book Computational Methods for Controller Design published by Springer.

Dr. Dahleh is interested in problems at the interface of robust control, filtering, information theory, and computation which include control problems with communication constraints and distributed mobile agents with local decision capabilities. He is also interested in various problems in network science including distributed computation over noisy network as well as information propagation over complex engineering and social networks. He is also interested in model reduction problems for discrete-alphabet hidden Markov models and universal learning approaches for systems with both continuous and discrete alphabets. He is also interested in the interface between systems theory and neurobiology, and in particular, in providing an anatomically consistent model of the motor control system. 